\title{
VARIATIONAL FORMULATION OF PROBLEMS INVOLVING FRACTIONAL ORDER DIFFERENTIAL OPERATORS
}

\author{
BANGTI JIN, RAYTCHO LAZAROV, JOSEPH PASCIAK, AND WILLIAM RUNDELL
}

\begin{abstract}
In this work, we consider boundary value problems involving either Caputo or Riemann-Liouville fractional derivatives of order $\alpha \in(1,2)$ on the unit interval $(0,1)$. These fractional derivatives lead to nonsymmetric boundary value problems, which are investigated from a variational point of view. The variational problem for the Riemann-Liouville case is coercive on the space $H_{0}^{\alpha / 2}(0,1)$ but the solutions are less regular, whereas that for the Caputo case involves different test and trial spaces. The numerical analysis of these problems requires the so-called shift theorems which show that the solutions of the variational problem are more regular. The regularity pickup enables one to establish convergence rates of the finite element approximations. The analytical theory is then applied to the Sturm-Liouville problem involving a fractional derivative in the leading term. Finally, extensive numerical results are presented to illustrate the error estimates for the source problem and eigenvalue problem.
\end{abstract}

\section{INTRODUCTION}

In this work we consider the following fractional-order source problem: find $u$ such that

$$
\begin{gathered}
-{ }_{0} D_{x}^{\alpha} u+q u=f \quad \text { in } D=(0,1), \\
u(0)=u(1)=0,
\end{gathered}
$$

where $\alpha \in(1,2)$ is the order of the derivative, and ${ }_{0} D_{x}^{\alpha}$ refers to either the left-sided Caputo or Riemann-Liouville fractional derivative of order $\alpha$ defined below in (2.1) and (2.2), respectively. Here $f$ is a function in $L^{2}(D)$ or other suitable Sobolev space. The potential coefficient $q \in L^{\infty}(D)$ is a bounded measurable function. Further, to illustrate the abstract theory, we consider the following fractional SturmLiouville problem (FSLP): find $u$ and $\lambda \in \mathbb{C}$ such that

$$
\begin{gathered}
-{ }_{0} D_{x}^{\alpha} u+q u=\lambda u \text { in } D, \\
u(0)=u(1)=0 .
\end{gathered}
$$

1.1. Motivation of the model (1.1). The interest in the model (1.1) is largely motivated by the studies on anomalous diffusion processes. Diffusion is one of the most prominent and ubiquitous transport mechanisms found in nature. At a microscopic level, it is the result of the random motion of individual particles,

Received by the editor August 20, 2013 and, in revised form, February 12, 2014 and April 6, 2014.

2010 Mathematics Subject Classification. Primary 65L60, 65N12, 65N30.

Key words and phrases. Fractional boundary value problem, Caputo derivative, RiemannLiouville derivative, variational formulation, finite element method, fractional Sturm-Liouville problem. 
and the use of the Laplace operator in the canonical diffusion model rests on a Brownian assumption on the particle motion. However, over the last two decades, a large body of literature has shown that anomalous diffusion, in which the mean square variance grows faster (superdiffusion) or slower (subdiffusion) than that in a Gaussian process, offers a superior fit to experimental data observed in a number of important practical applications, e.g., viscoelastic materials, subsurface flow and plasma physics.

The origin of non-Gaussian diffusion can be attributed to either the existence of long-range correlations in the dynamics or the presence of anomalously large particle jumps. The former describes subdiffusion and leads to a diffusion equation with a fractional derivative in time; see [23] and references therein for an extensive list of applications. The latter is suitable for modeling superdiffusion processes. It can be derived from the following observation at a microscopic level: the particle motion might be dependent, and can frequently take very large jumps, following some heavy-tailed probability distribution. The underlying stochastic process deviates significantly from the Brownian motion for the classical diffusion process. Instead, a Lévy flight is often adopted, where the probability distribution $p(\ell)$ of the particle jumps $\ell$ is broad in the sense that the variance $\operatorname{Var}_{p}[\ell]=\infty$. It is well known that in this case, the central limit theorem cannot be applied, and the limit distribution of the arithmetic mean, instead of being a Gaussian distribution, is an $\alpha$-stable Lévy distribution. At a macroscopic level, it gives rise to a spatial fractional diffusion equation [4,8]

$$
\frac{\partial u}{\partial t}-\mathbf{D}_{\theta}^{\alpha} u+q u=f
$$

In the model (1.3),$\theta \in[0,1]$ is a skewness parameter, and the differential operator $\mathbf{D}_{\theta}^{\alpha}$ is defined by

$$
\mathbf{D}_{\theta}^{\alpha} u=\theta{ }_{0}^{R} D_{x}^{\alpha} u+(1-\theta){ }_{x}^{R} D_{1}^{\alpha} u,
$$

where ${ }_{0}^{R} D_{x}^{\alpha}$ and ${ }_{x}^{R} D_{1}^{\alpha}$ denote the left- and right-sided Riemann-Liouville fractional derivatives, respectively. Physically, the fractional derivative $\mathbf{D}_{\theta}^{\alpha} u$ can also be interpreted as a nonlocal Fickian law [5].

The majority of experimental studies $[4,8$, on the model (1.3) focuses on the case $\theta=1 / 2$, which is suitable for symmetric processes, i.e., $p(\ell)=p(-\ell)$. However, in some physical systems, e.g., geophysical flows and magnetized plasmas [7, 33, asymmetric transport seems inherent. The extreme of asymmetric case is $\theta=0$ or $\theta=1$, and it has been analyzed in 9 . In some models the Riemann-Liouville derivative may cause a mass imbalance and hence it has been suggested to use the Caputo derivative to approximate the Riemann-Liouville derivative [31,38; see also [20]. Numerous experimental studies have convincingly demonstrated that the model (1.3) can provide faithful description of superdiffusion processes, and thus has attracted considerable attentions in practice. The model (1.1) represents the steady state of the model (1.3), and the choice of the fractional derivative rests on the asymmetric transport assumption (possibly also with regularization).

Apart from the preceding motivations from anomalous diffusion 20, the FSLP (1.2) also arises in M. Djrbashian's construction on certain spaces of analytic functions [10, Chapter 1], where the eigenfunctions of problems similar to (1.2) are used to construct a certain bi-orthogonal basis. This construction dates at least back to [12]; see also 24] for a related study. 
1.2. Relevant studies. Because of its extraordinary modeling capability, the accurate simulation of (1.3) has become imperative. Closed form solutions are available only in a few very limited cases, and hence a number of numerical methods, prominently the finite difference method, have been developed for the timedependent superdiffusion problem (1.3). In [35, a second-order finite difference scheme based on the Crank-Nicolson formula in time and the shifted Grünwald formula in space was proposed for the one-dimensional diffusion problem with a Riemann-Liouville derivative in space, and its stability, consistency and convergence was provided. See also [26, 34 for related works. In [32, Shkhanukov analyzed a finite difference scheme for a second-order differential equation with a fractional derivative in the lower-order term. However, the nonlocality of the fractional derivative leads to storage issues for these schemes. Recently, high-order finite difference schemes like the weighted and shifted Grünwald difference have received some interest, and the Grünwald formula on a uniform stencil leads to Toeplitz matrices, which can be exploited for space storage reduction and a fast solution via fast Fourier transform [36.

In contrast, the theoretical analysis on (1.1) remains scarce. This is attributed to the fact that it involves mathematical difficulties that are not present in the analysis of canonical second-order elliptic equations. In particular, the differential operator ${ }_{0} D_{x}^{\alpha}$ in (1.1) is nonlocal. In [14,15], a first rigorous analysis of the well-posedness of the weak formulation of the model (1.1) with a Riemann-Liouville derivative was presented via their relation to fractional-order Sobolev spaces. In [14] an optimal error estimate was also provided for the Galerkin finite element method under the assumption that the solution has full regularity, i.e., $\|u\|_{H^{\alpha}(D)} \leq c\|f\|_{L^{2}(D)}$. Unfortunately, such regularity is not justified in general (see Theorem 4.4 and Remark 4.2). Recently, Wang and Yang [37] generalized the analysis to the case of fractional-order derivatives involving a variable coefficient, analyzed the regularity of the solution in Hölder spaces, and established the well-posedness of a PetrovGalerkin formulation. However, the discrete inf-sup condition was not established and hence an error estimate of the discrete approximations was not provided.

The numerical analysis on the FSLP (1.2) is scarce. Al-Mdallal [2] applied the Adomian decomposition method to the FSLP with a Riemann-Liouville derivative. However, there is no mathematical analysis of the numerical scheme, and it can only locate one eigenvalue at a time. In [20, a Newton type method was proposed for computing eigenvalues to the FSLP with a Caputo derivative. However, since it involves many solves of (possibly stiff) fractional ordinary differential equations, the method is expensive. Hence, there seems to be no known fast, accurate and yet justified algorithm for the FSLP.

1.3. Our contributions. The goal of this work is to: (1) revisit the variational formulation for problem (1.1) for both cases of Riemann-Liouville and Caputo fractional derivatives, (2) establish the variational stability and shift theorems, (3) develop relevant finite element analysis and derive error estimates of the discrete approximations expressed in terms of the smoothness of the right-hand side only, and (4) illustrate the theoretical developments on the fractional Sturm-Liouville problem, to approximate multiple eigenvalues simultaneously with provable error estimates. We note that the Caputo case, which is very natural with its convenient treatment of boundary conditions, has not been previously considered in this 
context. There is an obvious difficulty in the attempt to extend the Caputo derivative operator defined below in (2.1) from functions in $C^{n}(D)$ to fractional order Sobolev spaces $H^{s}(D)$. In this paper we have developed a strategy that allows us to overcome this difficulty.

In brevity, the study of problem (1.1) from a variational point of view is as follows. The Riemann-Liouville case leads to a nonsymmetric but coercive bilinear form on the space $H_{0}^{\alpha / 2}(D)$; cf. Theorem 4.3. This fact for the case $q=0$ has been established earlier in 14. The Caputo case requires a test space $V$ that is different from the solution space $U$ and involves a nonlocal integral constraint. Further, we establish the well-posedness of the variational formulations, and more importantly, we establish regularity pickup of the weak solutions of (1.1) (cf. Theorems 4.4 and 4.7), and that of the weak solutions to the adjoint problems. We note that the solution regularity has only been assumed earlier in the error analysis of [14. The regularity pickup is essential for proving (optimal) convergence rates for the finite element method. Further, this technique is used for studying the finite element approximation of problem (1.2). The main theoretical result, i.e., error estimates for the eigenvalue approximations, is stated in Theorem 6.2 .

The rest of the paper is organized as follows. In Section 2 we recall preliminaries on fractional calculus, and study fractional derivatives as operators on fractional Sobolev spaces. Then in Section 3. we explicitly construct the strong solution representation via fractional-order integral operators. The continuous variational formulations are developed in Section 4 and their well-posedness is also established. Then in Section 5, the stability of the discrete variational formulations is studied, and error estimates of the finite element approximations are provided. In Section 6 , we illustrate the general theory with the Sturm-Liouville problem with a fractional derivative. Finally, in Section 7 some illustrative numerical results are presented to confirm the error estimates. Throughout, the notation $c$, with or without subscript, refers to a generic positive constant which can take different values at different occurrences, but it is always independent of the solution $u$ and the mesh size $h$, and $n$ denotes a fixed positive integer.

\section{Fractional differential operators on fractional Sobolev spaces}

First, we briefly recall two common fractional derivatives: the Caputo and Riemann-Liouville forms. For any positive noninteger real number $\beta$ with $n-1<$ $\beta<n$, the (formal) left-sided Caputo fractional derivative of order $\beta$ is defined by (see, e.g., [21, pp. 92], 25])

$$
{ }_{0}^{C} D_{x}^{\beta} u={ }_{0} I_{x}^{n-\beta}\left(\frac{d^{n} u}{d x^{n}}\right),
$$

and the (formal) left-sided Riemann-Liouville fractional derivative of order $\beta$ is defined by [21, pp. 70]:

$$
{ }_{0}^{R} D_{x}^{\beta} u=\frac{d^{n}}{d x^{n}}\left({ }_{0} I_{x}^{n-\beta} u\right) .
$$

In the definitions (2.1) and (2.2), ${ }_{0} I_{x}^{\gamma}$ for $\gamma>0$ is the left-sided Riemann-Liouville fractional integral operator of order $\gamma$ defined by

$$
{ }_{0} I_{x}^{\gamma} f=\frac{1}{\Gamma(\gamma)} \int_{0}^{x}(x-t)^{\gamma-1} f(t) d t
$$


where $\Gamma(\cdot)$ is Euler's Gamma function defined by $\Gamma(x)=\int_{0}^{\infty} t^{x-1} e^{-t} d t, x>0$. As the order $\gamma$ approaches 0 , we can identify the operator ${ }_{0} I_{x}^{\gamma}$ with the identity operator [25, pp. 65, eq. (2.89)]. The fractional integral operator ${ }_{0} I_{x}^{\gamma}$ satisfies a semigroup property, i.e., for $\gamma, \delta>0$ and smooth $u$, there holds [21, Lemma 2.3, pp. 73]

$$
{ }_{0} I_{x}^{\gamma+\delta} u={ }_{0} I_{x}^{\gamma}{ }_{0} I_{x}^{\delta} u
$$

This identity extends to the space $L^{2}(D)$ by a density argument.

Clearly, the fractional derivatives ${ }_{0}^{C} D_{x}^{\beta}$ and ${ }_{0}^{R} D_{x}^{\beta}$ are well defined for functions in $C^{n}[0,1]$ and are related to each other by the formula (cf. [21, pp. 91, eq. (2.4.6)])

$$
{ }_{0}^{C} D_{x}^{\beta} u={ }_{0}^{R} D_{x}^{\beta} u-\sum_{k=0}^{n-1} \frac{u^{(k)}(0)}{\Gamma(k-\beta+1)} x^{k-\beta} .
$$

This relation will be used to construct the solution representation in the Caputo case in Section 3 .

The right-sided versions of fractional-order integrals and derivatives are defined analogously, i.e.,

and

$$
{ }_{x} I_{1}^{\gamma} f=\frac{1}{\Gamma(\gamma)} \int_{x}^{1}(t-x)^{\gamma-1} f(t) d t
$$

$$
{ }_{x}^{C} D_{1}^{\beta} u=(-1)^{n}{ }_{x} I_{1}^{n-\beta}\left(\frac{d^{n} u}{d x^{n}}\right), \quad{ }_{x}^{R} D_{1}^{\beta} u=(-1)^{n} \frac{d^{n}}{d x^{n}}\left({ }_{x} I_{1}^{n-\beta} u\right) .
$$

The formula analogous to (2.4) is [21, pp. 91, eq. (2.4.7)]

$$
{ }_{x}^{C} D_{1}^{\beta} u={ }_{x}^{R} D_{1}^{\beta} u-\sum_{k=0}^{n-1} \frac{(-1)^{k} u^{(k)}(1)}{\Gamma(k-\beta+1)}(1-x)^{k-\beta} .
$$

Finally, we observe that for $\phi, \varphi \in L^{2}(D)$ and $\beta>-1$, there holds

$$
\begin{aligned}
\int_{0}^{1} \int_{0}^{x}(x-t)^{\beta}|\phi(t) \varphi(x)| d t d x & \leq \frac{1}{2} \int_{0}^{1} \int_{0}^{x}(x-t)^{\beta}\left[\phi(t)^{2}+\varphi(x)^{2}\right] d t d x \\
& \leq \frac{1}{2(1+\beta)}\left[\|\phi\|_{L^{2}(D)}^{2}+\|\varphi\|_{L^{2}(D)}^{2}\right] .
\end{aligned}
$$

Here the second line follows from the inequality $\int_{0}^{x}(x-t)^{\beta} d t \leq \int_{0}^{1}(1-t)^{\beta} d t=\frac{1}{1+\beta}$. Therefore, Fubini's Theorem yields the following useful change of integration order formula (cf. also [21, Lemma 2.7]):

$$
\left({ }_{0} I_{x}^{\beta} \phi, \varphi\right)=\left(\phi,{ }_{x} I_{1}^{\beta} \varphi\right), \quad \text { for all } \phi, \varphi \in L^{2}(D) .
$$

In order to investigate the model (1.1), we study these operators in fractional Sobolev spaces. This is motivated by the fact that the finite element method for usual second-order elliptic problems is most conveniently analyzed in Sobolev spaces and, in particular, optimal convergence rates with respect to the data regularity hinge essentially on the Sobolev regularity of the solutions. Analogously, the regularity properties of solutions to (1.1) are most naturally studied on fractional order Sobolev spaces. However, this requires the extension of the formal definition of the fractional derivatives and to this end we first introduce some function spaces.

For any $\beta \geq 0$, we denote $H^{\beta}(D)$ to be the Sobolev space of order $\beta$ on the unit interval $D$, and $\widetilde{H}^{\beta}(D)$ to be the set of functions in $H^{\beta}(D)$ whose extension by zero to $\mathbb{R}$ are in $H^{\beta}(\mathbb{R})$. These spaces are characterized in [18. For example, it is 
known that for $\beta \in(0,1)$, the space $\widetilde{H}^{\beta}(D)$ coincides with the interpolation space $\left[L^{2}(D), H_{0}^{1}(D)\right]_{\beta}$. It is important for our further study to note that $\phi \in \widetilde{H}^{\beta}(D)$, $\beta>3 / 2$, satisfies the boundary conditions $\phi(0)=\phi^{\prime}(0)=0$ and $\phi(1)=\phi^{\prime}(1)=0$. Analogously, we define $\widetilde{H}_{L}^{\beta}(D)$ (respectively, $\widetilde{H}_{R}^{\beta}(D)$ ) to be the set of functions $u$ whose extension by zero $\tilde{u}$ are in $H^{\beta}(-\infty, 1)$ (respectively, $\left.H^{\beta}(0, \infty)\right)$. Here for $u \in \widetilde{H}_{L}^{\beta}(D)$, we set $\|u\|_{\widetilde{H}_{L}^{\beta}(D)}:=\|\tilde{u}\|_{H^{\beta}(-\infty, 1)}$ with the analogous definition for the norm in $\widetilde{H}_{R}^{\beta}(D)$. Let $\widetilde{C}_{L}^{\beta}(D)$ (respectively, $\widetilde{C}_{R}^{\beta}(D)$ ) denote the set of functions in $v \in C^{\infty}[0,1]$ satisfying $v(0)=v^{\prime}(0)=\ldots=v^{(k)}(0)=0$ (respectively, $v(1)=$ $\left.v^{\prime}(1)=\ldots=v^{(k)}(1)=0\right)$ for any nonnegative integer $k<\beta-1 / 2$. It is not hard to see that the spaces $\widetilde{C}_{L}^{\beta}(D)$ and $\widetilde{C}_{R}^{\beta}(D)$ are dense in the spaces $\widetilde{H}_{L}^{\beta}(D)$ and $\widetilde{H}_{R}^{\beta}(D)$, respectively. Throughout, we denote by $\tilde{u}$ the extension of $u$ by zero to $\mathbb{R}$.

The first result forms the basis for our investigation of fractional derivatives in Sobolev spaces. It was shown in [14, Lemma 2.6], but we provide an alternative proof since the idea will be used later.

Theorem 2.1. For any $\beta \in(n-1, n)$, the operators ${ }_{0}^{R} D_{x}^{\beta} u$ and ${ }_{x}^{R} D_{1}^{\beta} u$ defined for $u \in C_{0}^{\infty}(D)$ extend continuously to operators (still denoted by ${ }_{0}^{R} D_{x}^{\beta} u$ and ${ }_{x}^{R} D_{1}^{\beta} u$ ) from $\widetilde{H}^{\beta}(D)$ to $L^{2}(D)$.

Proof. We first consider the left-sided case. For $v \in C_{0}^{\infty}(\mathbb{R})$, we define

$$
{ }_{-\infty}^{R} D_{x}^{\beta} v=\frac{d^{n}}{d x^{n}}\left(\int_{-\infty}^{x}(x-t)^{\beta-n} v(t) d t\right) .
$$

We note the following identity for the Fourier transform

$$
\mathcal{F}\left({ }_{-\infty}^{R} D_{x}^{\beta} v\right)(\omega)=(-i \omega)^{\beta} \mathcal{F}(v)(\omega), \quad \text { i.e., } \quad{ }_{-\infty}^{R} D_{x}^{\beta} v(x)=\mathcal{F}^{-1}\left((-i \omega)^{\beta} \mathcal{F}(v)(\omega)\right),
$$

which holds for $v \in C_{0}^{\infty}(\mathbb{R})$ (cf. [25, pp. 112, eq. (2.272)] or [21, pp. 90, eq. (2.3.27)]). It follows from Plancherel's theorem that

$$
\left\|{ }_{-\infty}^{R} D_{x}^{\beta} v\right\|_{L^{2}(\mathbb{R})}=\left\|\mathcal{F}\left({ }_{-\infty}^{R} D_{x}^{\beta} v\right)\right\|_{L^{2}(\mathbb{R})} \leq c\|v\|_{H^{\beta}(\mathbb{R})} .
$$

Thus, we can continuously extend ${ }_{-\infty}^{R} D_{x}^{\beta}$ to an operator from $H^{\beta}(\mathbb{R})$ into $L^{2}(\mathbb{R})$ by formula (2.8).

We note that for $u \in C_{0}^{\infty}(D)$, there holds

$$
{ }_{0}^{R} D_{x}^{\beta} u=\left.{ }_{-\infty}^{R} D_{x}^{\beta} \tilde{u}\right|_{D} .
$$

By definition, $u \in \widetilde{H}^{\beta}(D)$ implies that $\tilde{u}$ is in $H^{\beta}(\mathbb{R})$ and hence

$$
\left\|{ }_{-\infty}^{R} D_{x}^{\beta} \tilde{u}\right\|_{L^{2}(\mathbb{R})} \leq c\|u\|_{\widetilde{H}^{\beta}(D)} .
$$

Thus, formula (2.9) provides an extension of the operator ${ }_{0}^{R} D_{x}^{\beta}$ defined on $C_{0}^{\infty}(D)$ to a bounded operator from the space $\widetilde{H}^{\beta}(D)$ into $L^{2}(D)$.

The right-sided derivative case is essentially identical except for replacing (2.7) and (2.8) with

and

$$
{ }_{x}^{R} D_{\infty}^{\beta} v=(-1)^{n} \frac{d^{n}}{d x^{n}}\left(\int_{x}^{\infty}(t-x)^{\beta-n} v(t) d t\right)
$$

$$
\mathcal{F}\left({ }_{x}^{R} D_{\infty}^{\beta} v\right)(\omega)=(i \omega)^{\beta} \mathcal{F}(v)(\omega)
$$

This completes the proof of the theorem. 
Remark 2.1. We clearly have ${ }_{-\infty}^{R} D_{x}^{\beta} v(x)=0$ for $x<1$ when $v \in \widetilde{C}_{L}^{\beta}(0,2)$ is supported on the interval $[1,2]$. By a density argument this also holds for $v \in$ $\widetilde{H}_{L}^{\beta}(0,2)$ supported on $[1,2]$.

The next result slightly relaxes the condition in Theorem 2.1.

Theorem 2.2. For $\beta \in(n-1, n)$, the operator ${ }_{0}^{R} D_{x}^{\beta} u$ defined for $u \in \widetilde{C}_{L}^{\beta}(D)$ extends continuously to an operator from $\widetilde{H}_{L}^{\beta}(D)$ to $L^{2}(D)$. Similarly, the operator ${ }_{x}^{R} D_{1}^{\beta}$ defined for $u \in \widetilde{C}_{R}^{\beta}(D)$ extends continuously to an operator from $\widetilde{H}_{R}^{\beta}(D)$ to $L^{2}(D)$.

Proof. We only prove the result for the left-sided derivative since the proof for the right-sided derivative case is identical. For a given $u \in \widetilde{H}_{L}^{\beta}(D)$, we let $u_{0}$ be a bounded extension of $u$ to $\widetilde{H}^{\beta}(0,2)$, and then set

$$
{ }_{0}^{R} D_{x}^{\beta} u=\left.{ }_{0}^{R} D_{x}^{\beta} \tilde{u}_{0}\right|_{D}
$$

It is a direct consequence of Remark 2.1 that ${ }_{0}^{R} D_{x}^{\beta} u$ is independent of the extension $u_{0}$ and coincides with the formal definition of ${ }_{0}^{R} D_{x}^{\beta} u$ when $u \in \widetilde{C}_{L}^{\beta}(D)$. We obviously have

$$
\left\|_{0}^{R} D_{x}^{\beta} u\right\|_{L^{2}(D)} \leq\left\|{ }_{-\infty}^{R} D_{x}^{\beta} \tilde{u}_{0}\right\|_{L^{2}(\mathbb{R})} \leq c\|u\|_{\widetilde{H}_{L}^{\beta}(D)} .
$$

This completes the proof.

Remark 2.2. Even though ${ }_{0}^{R} D_{x}^{\beta}$ makes sense as an operator from $\widetilde{H}_{L}^{\beta}(D)$ into $L^{2}(D)$, it apparently cannot be extended to useful larger spaces, for example, ${ }_{0}^{R} D_{x}^{\beta}(1)=$ $c_{\beta} x^{-\beta}$ is not in the space $L^{2}(D)$, if $\beta>\frac{1}{2}$. Accordingly, it is not obvious in what space one should seek the solutions of problem (1.1) with a Riemann-Liouville derivative and in case of a nonzero Dirichlet boundary condition at $x=0$. However, it is clear that, at least for $\alpha \in(3 / 2,2)$, the solution is not generally in $\widetilde{H}^{\alpha}(D)$ since such functions satisfy the additional boundary conditions $u^{\prime}(0)=u^{\prime}(1)=0$.

Remark 2.3. Lemma 4.1 below shows that for $\beta \in(0,1)$, the operators ${ }_{0}^{C} D_{x}^{\beta}$ and ${ }_{0}^{R} D_{x}^{\beta}$ coincide on the space $\widetilde{H}_{L}^{1}(D)$. Thus, for $\beta \in(1 / 2,1)$, the continuous extension of ${ }_{0}^{C} D_{x}^{\beta}$ defined on $\widetilde{C}_{L}^{\beta}(D)$ to $\widetilde{H}_{L}^{1}(D)$ is ${ }_{0}^{C} D_{x}^{\beta} \equiv{ }_{0}^{R} D_{x}^{\beta}$. However, the extension of ${ }_{0}^{C} D_{x}^{\beta}$ to fractional order Sobolev spaces $\widetilde{H}_{L}^{\beta}(D)$ remains elusive.

\section{Strong SOlUtions of FraCtional ORDER DifFEREnTial EQUATIONS}

The problem we now face is to make some sense out of the model (1.1). We start by studying the case of $q=0$. We shall construct functions whose fractional derivatives make sense and satisfy (1.1). The following smoothing property of the fractional integral operators ${ }_{0} I_{x}^{\beta}$ and ${ }_{x} I_{1}^{\beta}$ will play a central role in the construction. We note that the smoothing property of the operator ${ }_{0} I_{x}^{\beta}$ in $L^{p}(D)$ spaces and Hölder spaces was studied extensively in [28, Chapter 1, §3].

Theorem 3.1. For any $s, \beta \geq 0$, the operators ${ }_{0} I_{x}^{\beta}$ and ${ }_{x} I_{1}^{\beta}$ are bounded maps from $\widetilde{H}^{s}(D)$ into $\widetilde{H}_{L}^{s+\beta}(D)$ and $\widetilde{H}_{R}^{s+\beta}(D)$, respectively.

Proof. The key idea of the proof is to extend $f \in \widetilde{H}^{s}(D)$ to a function $\widetilde{f} \in \widetilde{H}^{s}(0,2)$ whose moments up to $(k-1)$ th order vanish with $k>\beta-1 / 2$. To this end, we 
employ orthogonal polynomials $\left\{p_{0}, p_{1}, \ldots, p_{k-1}\right\}$ with respect to the inner product $\langle\cdot, \cdot\rangle$ defined by

$$
\langle u, v\rangle=\int_{1}^{2}((x-1)(2-x))^{l} u(x) v(x) d x,
$$

where the integer $l$ satisfies $l>s-1 / 2$ so that

$$
((x-1)(2-x))^{l} p_{i} \in \widetilde{H}^{s}(1,2), \quad i=0, \ldots, k-1 .
$$

Then we set $w_{j}=\gamma_{j}((x-1)(2-x))^{l} p_{j}$ with $\gamma_{j}$ chosen so that

$$
\int_{1}^{2} w_{j} p_{j} d x=1 \quad \text { so that } \quad \int_{1}^{2} w_{j} p_{l} d x=\delta_{j, l}, \quad j, l=0, \ldots, k-1 .
$$

Next we extend both $f$ and $w_{j}, j=0, \ldots, k-1$ by zero to $(0,2)$ by setting

$$
f_{e}=f-\sum_{j=0}^{k-1}\left(\int_{0}^{1} f p_{j} d x\right) w_{j}
$$

The resulting function $f_{e}$ has vanishing moments for $j=0, \ldots, k-1$ and by construction it is in the space $\widetilde{H}^{s}(0,2)$. Further, obviously there holds the inequality $\left\|f_{e}\right\|_{L^{2}(0,2)} \leq C\|f\|_{L^{2}(D)}$, i.e., the extension is bounded in $L^{2}(D)$. As usual, we denote by $\tilde{f}_{e}$ the extension of $f_{e}$ to $\mathbb{R}$ by zero.

Now for $x \in(0,1)$, there holds $\left({ }_{0} I_{x}^{\beta} f\right)(x)=\left({ }_{-\infty} I_{x}^{\beta} \widetilde{f}_{e}\right)(x)$, where

$$
-\infty I_{x}^{\beta}\left(\tilde{f}_{e}\right)=\frac{1}{\Gamma(\beta)} \int_{-\infty}^{x}(x-t)^{\beta-1} \widetilde{f}_{e}(t) d t .
$$

Meanwhile, we have (see [25, pp. 112, eq. (2.272)] or [21, pp. 90, eq. (2.3.27)])

$$
\mathcal{F}\left(-\infty I_{x}^{\beta} \widetilde{f}_{e}\right)(\omega)=(-i \omega)^{-\beta} \mathcal{F}\left(\tilde{f}_{e}\right)(\omega)
$$

and hence by Plancherel's theorem

$$
\left\|_{-\infty} I_{x}^{\beta} \widetilde{f}_{e}\right\|_{L^{2}(\mathbb{R})}^{2}=\int_{\mathbb{R}}|\omega|^{-2 \beta}\left|\mathcal{F}\left(\widetilde{f}_{e}\right)(\omega)\right|^{2} d \omega .
$$

We note that by a Taylor expansion centered at 0 , there holds

$$
\begin{aligned}
e^{-i \omega x} & -1-(-i \omega) x-\cdots-\frac{(-i \omega)^{k-1} x^{k-1}}{(k-1) !} \\
& =\frac{(-i \omega x)^{k}}{k !}+\frac{(-i \omega x)^{k+1}}{(k+1) !}+\frac{(-i \omega x)^{k+2}}{(k+2) !}+\cdots=(-i \omega)^{k}{ }_{0} I_{x}^{k}\left(e^{-i \omega x}\right) .
\end{aligned}
$$

Clearly, there holds $\left|{ }_{0} I_{x}^{k}\left(e^{-i \omega x}\right)\right| \leq\left|{ }_{0} I_{x}^{k}(1)\right|=\frac{x^{k}}{k !}$. Since the first $k$ moments of $\tilde{f}_{e}$ vanish, multiplying the above identity by $\widetilde{f}_{e}$ and integrating over $\mathbb{R}$ gives

$$
\mathcal{F}\left(\tilde{f}_{e}\right)(\omega)=(-i \omega)^{k} \frac{1}{\sqrt{2 \pi}} \int_{\mathbb{R}}{ }_{0} I_{x}^{k}\left(e^{-i \omega x}\right) \tilde{f}_{e}(x) d x,
$$

and upon noting $\operatorname{supp}\left(\widetilde{f}_{e}\right) \subset(0,2)$, consequently,

$$
\left|\mathcal{F}\left(\widetilde{f}_{e}\right)(\omega)\right| \leq \frac{2^{k}}{\sqrt{\pi} k !}|\omega|^{k}\left\|f_{e}\right\|_{L^{2}(0,2)} \leq c|\omega|^{k}\|f\|_{L^{2}(D)} .
$$


We then have

$$
\begin{aligned}
\left\|_{-\infty} I_{x}^{\beta} \widetilde{f}_{e}\right\|_{H^{\beta+s}(\mathbb{R})}^{2} & =\int_{\mathbb{R}}\left(1+|\omega|^{2}\right)^{\beta+s}|\omega|^{-2 \beta}\left|\mathcal{F}\left(\tilde{f}_{e}\right)\right|^{2} d \omega \\
& \leq c_{1}\|f\|_{L^{2}(D)} \int_{|\omega|<1}|\omega|^{-2 \beta+2 k} d \omega+c_{2} \int_{|\omega|>1}|\omega|^{2 s}\left|\mathcal{F}\left(\widetilde{f}_{e}\right)\right|^{2} d \omega \\
& \leq c\|f\|_{\widetilde{H}^{s}(D)} .
\end{aligned}
$$

The desired assertion follows from this and the trivial inequality $\left\|_{0} I_{x}^{\beta} f\right\|_{H^{\beta+s}(D)} \leq$ $\left\|-\infty I_{x}^{\beta} \widetilde{f}_{e}\right\|_{H^{\beta+s}(\mathbb{R})}^{2}$.

Remark 3.1. By means of the extension in Theorem 2.2 the operator ${ }_{0} I_{x}^{\beta}$ is bounded from $\widetilde{H}_{L}^{s}(D)$ to $\widetilde{H}_{L}^{\beta+s}(D)$, and ${ }_{x} I_{1}^{\beta}$ is bounded from $\widetilde{H}_{R}^{s}(D)$ to $\widetilde{H}_{R}^{\beta+s}(D)$.

A direct consequence of Theorem 3.1 is the following useful corollary.

Corollary 3.2. Let $\gamma$ be nonnegative. Then the functions $x^{\gamma}$ and $(1-x)^{\gamma}$ belong to $\widetilde{H}_{L}^{\beta}(D)$ and $\widetilde{H}_{R}^{\beta}(D)$, respectively, for any $0 \leq \beta<\gamma+1 / 2$.

Proof. We note the relations $x^{\gamma}=c_{\gamma}{ }_{0} I_{x}^{\gamma}(1)$ and $(1-x)^{\gamma}=c_{\gamma x} I_{1}^{\gamma}(1)$. The desired result follows from Theorem 3.1 and the fact that $1 \in \widetilde{H}_{L}^{\delta}(D)$ and $1 \in \widetilde{H}_{R}^{\delta}(D)$ for any $\delta \in[0,1 / 2)$.

Now we are in a position to construct the strong solutions to problem (1.1) in the case of a vanishing potential, i.e., $q=0$. We first consider the Riemann-Liouville case. Here we fix $f \in L^{2}(D)$ and set $g={ }_{0} I_{x}^{\alpha} f \in \widetilde{H}_{L}^{\alpha}(D)$. By Theorem 2.2, the fractional derivative ${ }_{0}^{R} D_{x}^{\alpha} g$ is well defined. Now in view of the semigroup property (2.3), we deduce

$$
{ }_{0} I_{x}^{2-\alpha} g={ }_{0} I_{x}^{2-\alpha}{ }_{0} I_{x}^{\alpha} f={ }_{0} I_{x}^{2} f \in \widetilde{H}_{L}^{2}(D) .
$$

It is straightforward to check that $\left({ }_{0} I_{x}^{2} f\right)^{\prime \prime}=f$ holds for smooth $f$ and hence also for $f \in L^{2}(D)$ by a density argument. This yields ${ }_{0}^{R} D_{x}^{\alpha} g=f$. (This relation is reminiscent of the fundamental theorem in calculus.) We thus find that

$$
u=-{ }_{0} I_{x}^{\alpha} f+\left({ }_{0} I_{x}^{\alpha} f\right)(1) x^{\alpha-1}
$$

is a solution of problem (1.1) in the Riemann-Louiville case when $q=0$ since $u$ satisfies the correct boundary condition and ${ }_{0}^{R} D_{x}^{\alpha} x^{\alpha-1}=\left(c_{\alpha} x\right)^{\prime \prime}=0$.

Next we consider the Caputo case. To this end, we choose $s \geq 0$ so that $\alpha+$ $s \in(3 / 2,2)$. For smooth $u$ and $\alpha \in(1,2)$, the Caputo and Riemann-Liouville derivatives are related by (cf. (2.4) $)$

$$
{ }_{0}^{C} D_{x}^{\alpha} u={ }_{0}^{R} D_{x}^{\alpha} u-\frac{u(0)}{\Gamma(1-\alpha)} x^{-\alpha}-\frac{u^{\prime}(0)}{\Gamma(2-\alpha)} x^{1-\alpha} .
$$

Applying this formula to $g={ }_{0} I_{x}^{\alpha} f$ for $f \in \widetilde{H}^{s}(D)$ (in this case $g$ is in $\widetilde{H}_{L}^{\alpha+s}(D)$ by Theorem 3.1 and hence satisfies $g(0)=g^{\prime}(0)=0$ ) shows that ${ }_{0}^{C} D_{x}^{\alpha} g$ makes sense and equals ${ }_{0}^{R} D_{x}^{\alpha} g=f$. Thus a solution $u$ of problem (1.1) in the Caputo case with $q=0$ is given by

$$
u=-{ }_{0} I_{x}^{\alpha} f+\left({ }_{0} I_{x}^{\alpha} f\right)(1) x .
$$

Remark 3.2. The solution representation of the model (1.1) with a Caputo fractional derivative ${ }_{0}^{C} D_{x}^{\alpha} u$ and low-regularity source term $f \in \widetilde{H^{s}}(D)$ such that $\alpha+s \leq 3 / 2$ remains unclear. 
4. VARiational FORMUlations of the FRACTIONAL DERIVATIVE PROBLEMS

In this section we develop variational formulations of problem (1.1) and establish shift theorems for the variational solution. The shift theorems show the regularity pickup for the variational solution, which will be essential for deriving error estimates in Section 5. We shall first consider the case $q=0$ where we have an explicit representation of the solutions, and then the case of a general $q \neq 0$.

4.1. Derivation of the variational formulations. The starting point of the derivation of the variational formulations is the following lemma.

Lemma 4.1. For $u \in \widetilde{H}_{L}^{1}(D)$ and $\beta \in(0,1)$,

$$
{ }_{0}^{R} D_{x}^{\beta} u={ }_{0} I_{x}^{1-\beta}\left(u^{\prime}\right) .
$$

Similarly, for $u \in \widetilde{H}_{R}^{1}(D)$ and $\beta \in(0,1)$,

$$
{ }_{x}^{R} D_{1}^{\beta} u=-{ }_{x} I_{1}^{1-\beta}\left(u^{\prime}\right) .
$$

Proof. It suffices to prove the result for the left-sided derivative ${ }_{0}^{R} D_{x}^{\beta}$ since the rightsided case is analogous. For $u \in \widetilde{C}_{L}^{\beta}(D)$, (2.4) implies that

$$
{ }_{0}^{R} D_{x}^{\beta} u={ }_{0}^{C} D_{x}^{\beta} u={ }_{0} I_{x}^{1-\beta}\left(u^{\prime}\right) .
$$

Theorem 2.2 implies that the left-hand side extends to a continuous operator on $\widetilde{H}_{L}^{\beta}(D)$ and hence $\widetilde{H}_{L}^{1}(D)$ into $L^{2}(D)$. Meanwhile, Theorem 3.1 implies that the right-hand side of (4.1) extends to a bounded operator from $H^{1}(D)$ into $L^{2}(D)$. The lemma now follows by a density argument.

We next motivate the variational formulation in the Riemann-Liouville case. Upon taking $u$ as in (3.3), $g={ }_{0} I_{x}^{\alpha} f \in \widetilde{H}_{L}^{\alpha}(D)$ and $v \in C_{0}^{\infty}(D)$, Lemma 4.1 implies

$$
\begin{aligned}
\left({ }_{0}^{R} D_{x}^{\alpha} u, v\right) & =-\left(\left({ }_{0} I_{x}^{2-\alpha} g\right)^{\prime \prime}, v\right)=\left(\left({ }_{0} I_{x}^{2-\alpha} g\right)^{\prime}, v^{\prime}\right) \\
& =\left({ }_{0}^{R} D_{x}^{\alpha-1} g, v^{\prime}\right)=\left({ }_{0} I_{x}^{2-\alpha} g^{\prime}, v^{\prime}\right),
\end{aligned}
$$

where we have used the identity ${ }_{0}^{R} D_{x}^{\alpha} x^{\alpha-1}=0$ in the first step. Now the semigroup property (2.3) and the change of integration order formula (2.6) yield

$$
\left({ }_{0}^{R} D_{x}^{\alpha} u, v\right)=\left({ }_{0} I_{x}^{1-\alpha / 2} g^{\prime},{ }_{x} I_{1}^{1-\alpha / 2} v^{\prime}\right) .
$$

Since $g \in \widetilde{H}_{L}^{\alpha}(D)$ and $v \in \widetilde{H}^{1}(D)$, we can apply Lemma 4.1 again to conclude

$$
\left({ }_{0}^{R} D_{x}^{\alpha} u, v\right)=-\left({ }_{0}^{R} D_{x}^{\alpha / 2} g,{ }_{x}^{R} D_{1}^{\alpha / 2} v\right) .
$$

Further, by noting the identity ${ }_{0}^{R} D_{x}^{\alpha / 2} x^{\alpha-1}=c_{\alpha} x^{\alpha / 2-1} \in L^{2}(D)$ and $v^{\prime} \in L^{2}(D)$ from the assumption $v \in \widetilde{H}^{1}(D)$, we can apply the formula (2.6) to deduce

$$
\begin{aligned}
\left({ }_{0}^{R} D_{x}^{\alpha / 2} x^{\alpha-1},{ }_{x}^{R} D_{1}^{\alpha / 2} v\right) & =\left(c_{\alpha} x^{\alpha / 2-1},{ }_{x} I_{1}^{1-\alpha / 2} v^{\prime}\right) \\
& =\left(c_{\alpha 0} I_{x}^{1-\alpha / 2} x^{\alpha / 2-1}, v^{\prime}\right)=c_{\alpha}^{\prime}\left(1, v^{\prime}\right)=0,
\end{aligned}
$$

in view of the fact $v \in \widetilde{H}^{1}(D)$. Consequently,

$$
A(u, v):=-\left({ }_{0}^{R} D_{x}^{\alpha} u, v\right)=-\left({ }_{0}^{R} D_{x}^{\alpha / 2} u,{ }_{x}^{R} D_{1}^{\alpha / 2} v\right) .
$$


Later in Lemma 4.2, we shall show that $A(u, v)$ is a coercive and bounded bilinear form on the space $\widetilde{H}^{\alpha / 2}(D)$, and thus our variational formulation of problem (1.1) (with $q=0$ ) in the Riemann-Liouville case is to find $u \in U:=\widetilde{H}^{\alpha / 2}(D)$ satisfying

$$
A(u, v)=(f, v), \quad \text { for all } v \in U .
$$

We next consider the Caputo case. Following the definition of the solution in (3.4) in Section 3, we choose $\beta \geq 0$ so that $\alpha+\beta \in(3 / 2,2)$ and $f \in \widetilde{H}^{\beta}(D)$. By Theorem 3.1 the function $g={ }_{0} I_{x}^{\alpha} f$ lies in $\widetilde{H}_{L}^{\alpha+\beta}(D)$ and hence $g^{\prime}(0)=0$. Differentiating both sides of (3.4) and setting $x=0$ yields the identity $u^{\prime}(0)=$ $\left({ }_{0} I_{x}^{\alpha} f\right)(1)$, and thus, the solution representation (3.4) can be rewritten as

$$
u=-{ }_{0} I_{x}^{\alpha} f+x u^{\prime}(0):=-g(x)+x u^{\prime}(0) .
$$

Meanwhile, for $v \in \widetilde{C}_{R}^{\alpha / 2}(D)$, 4.2) implies

$$
-\left({ }_{0}^{R} D_{x}^{\alpha} g, v\right)=\left(\left({ }_{0} I_{x}^{2-\alpha} u\right)^{\prime \prime}, v\right)=\left(\left({ }_{0} I_{x}^{2-\alpha} g\right)^{\prime}, v^{\prime}\right),
$$

where we have again used the relation $g^{\prime}(0)=0$ in the last step. Analogous to the derivation of (4.3) and the identities ${ }_{0}^{C} D_{x}^{\alpha} g={ }_{0}^{R} D_{x}^{\alpha} g$ and ${ }_{0}^{C} D_{x}^{\alpha} x=0$, we then have

$$
-\left({ }_{0}^{C} D_{x}^{\alpha} u, v\right)=\left({ }_{0}^{R} D_{x}^{\alpha} g, v\right)=-A(g, v)=A(u, v)-u^{\prime}(0) A(x, v) .
$$

The term involving $u^{\prime}(0)$ cannot appear in a variational formulation in $\widetilde{H}^{\alpha / 2}(D)$. To circumvent this issue, we reverse the preceding steps and arrive at

$$
A(x, v)=\left({ }_{0} I_{x}^{2-\alpha} 1, v^{\prime}\right)=(\Gamma(3-\alpha))^{-1}\left(x^{2-\alpha}, v^{\prime}\right)=-(\Gamma(2-\alpha))^{-1}\left(x^{1-\alpha}, v\right) .
$$

Hence, in order to get rid of the troublesome term $-u^{\prime}(0) A(x, v)$ in (4.5), we require our test functions to satisfy the integral condition $\left(x^{1-\alpha}, v\right)=0$. Thus the variational formulation of (1.1) in the Caputo case (with $q=0$ ) is to find $u \in U$ satisfying

$$
A(u, v)=(f, v), \quad \text { for all } v \in V,
$$

with the test space

$$
V=\left\{\phi \in \widetilde{H}_{R}^{\alpha / 2}(D):\left(x^{1-\alpha}, \phi\right)=0\right\} .
$$

We shall show that this is a well-posed problem with a unique solution (for any $\left.f \in L^{2}(D)\right)$.

In the rest of this section, we discuss the stability of the variational formulations. Throughout, we denote by $U^{*}$ (respectively $V^{*}$ ) the set of bounded linear functionals on $U$ (respectively $V$ ), and slightly abuse $\langle\cdot, \cdot\rangle$ for duality pairing between $U$ and $U^{*}$ (or between $V$ and $V^{*}$ ). Further, we will denote by $\|\cdot\|_{U}$ the norm on the space $U$, etc.

Remark 4.1. We have seen that when $f$ is in $\widetilde{H}^{s}(D)$, with $\alpha+s>3 / 2$, then the solution $u$ constructed by (3.4) satisfies the variational equation and hence coincides with the unique solution to the variational equation. This may not be the case when $f$ is only in $L^{2}(D)$. Indeed, for $\alpha$ in $(1,3 / 2)$, the function $f=x^{1-\alpha}$ is in $L^{2}(D)$. However, the variational solution (with $q=0$ ) in this case is $u=0$ and clearly does not satisfy the strong-form differential equation ${ }_{0}^{C} D_{x}^{\alpha} u=f$. 
4.2. Variational stability in the Riemann-Liouville case. We now establish the stability of the variational formulations. The following lemma implies the variational stability in the Riemann-Liouville case with $q=0$. The result is well known [14], and the proof is provided only for completeness.

Lemma 4.2. Let $\alpha$ be in $(1,2)$. Then there is a positive constant $c=c(\alpha)$ satisfying

$$
c\|u\|_{\widetilde{H}^{\alpha / 2}(D)}^{2} \leq-\left({ }_{0}^{R} D_{x}^{\alpha / 2} u,{ }_{x}^{R} D_{1}^{\alpha / 2} u\right)=A(u, u), \quad \text { for all } u \in \widetilde{H}^{\alpha / 2}(D) .
$$

Proof. As in the proof of Theorem 2.1, we find for $u \in C_{0}^{\infty}(D)$,

$$
\begin{aligned}
-\left({ }_{0}^{R} D_{x}^{\alpha / 2} u,{ }_{x}^{R} D_{1}^{\alpha / 2} u\right) & =-\int_{-\infty}^{\infty}(i \omega)^{\alpha}|\mathcal{F}(\tilde{u})|^{2} d \omega=2 \cos \left(\left(1-\frac{\alpha}{2}\right) \pi\right) \int_{0}^{\infty} \omega^{\alpha}|\mathcal{F}(\tilde{u})|^{2} d \omega \\
& =\cos \left(\left(1-\frac{\alpha}{2}\right) \pi\right) \int_{-\infty}^{\infty}|\omega|^{\alpha}|\mathcal{F}(\tilde{u})|^{2} d \omega .
\end{aligned}
$$

Now suppose that there does not exist a constant satisfying (4.7). Then by the compact embedding of $\widetilde{H}^{\alpha / 2}(D)$ into $L^{2}(D)$, there is a sequence $\left\{u_{j}\right\} \subset \widetilde{H}^{\alpha / 2}(D)$ with $\left\|u_{j}\right\|_{\widetilde{H}^{\alpha / 2}(D)}=1$ convergent to some $u \in L^{2}(D)$ and satisfying

$$
\left\|u_{j}\right\|_{\widetilde{H}^{\alpha / 2}(D)}^{2}>-j\left({ }_{0}^{R} D_{x}^{\alpha / 2} u_{j},{ }_{x}^{R} D_{1}^{\alpha / 2} u_{j}\right) .
$$

It follows from (4.8) and (4.9), the sequence $\left\{\mathcal{F}\left(\tilde{u}_{j}\right)\right\}$ converges to zero in $\int_{-\infty}^{\infty}|\omega|^{\alpha} \mid$. $\left.\right|^{2} d \omega$, and by the convergence of the sequence $\left\{u_{j}\right\}$ in $L^{2}(D)$ and Plancherel's theorem, $\left\{\mathcal{F}\left(\tilde{u}_{j}\right)\right\}$ converges in $\int_{-\infty}^{\infty}|\cdot|^{2} d \omega$. Hence by the inequality $(1+s)^{p} \leq 1+s^{p}$ for $s \geq 0$ and $p \in[0,1],\left\{\mathcal{F}\left(\tilde{u}_{j}\right)\right\}$ is a Cauchy sequence and hence converges to $\mathcal{F}(\tilde{u})$ in the norm $\left(\int_{-\infty}^{\infty}\left(1+\omega^{2}\right)^{\alpha / 2}|\cdot|^{2} d \omega\right)^{1 / 2}$. This implies that $u_{j}$ converges to $u$ in $\widetilde{H}^{\alpha / 2}(D)$. Now Theorem 2.1 and (4.9) imply $-\left({ }_{0}^{R} D_{x}^{\alpha / 2} u,{ }_{x}^{R} D_{1}^{\alpha / 2} u\right)=0$, from which it follows that $\mathcal{F}(\tilde{u})=\tilde{u}=0$. This contradicts the assumption $\left\|u_{j}\right\|_{\widetilde{H}^{\alpha / 2}(D)}=1$ and completes the proof.

We now return to the problem with $q \neq 0$ and define

$$
a(u, v)=A(u, v)+(q u, v) .
$$

To this end, we make the following uniqueness assumption on the bilinear form.

Assumption 4.1. Let the bilinear form $a(u, v)$ with $u, v \in U$ satisfy:

(a) The problem of finding $u \in U$ such that $a(u, v)=0$ for all $v \in U$ has only the trivial solution $u \equiv 0$.

$\left(\mathrm{a}^{*}\right)$ The problem of finding $v \in U$ such that $a(u, v)=0$ for all $u \in U$ has only the trivial solution $v \equiv 0$.

We then have the following existence result.

Theorem 4.3 (Riemann-Liouville derivative). Let Assumption 4.1 hold and $q \in$ $L^{\infty}(D)$. Then for any given $F \in U^{*}$, there exists a unique solution $u \in U$ solving

$$
a(u, v)=\langle F, v\rangle, \quad \text { for all } v \in U .
$$

Proof. The proof is an application of the Petree-Tartar lemma [13, pp. 469, Lemma A.38]. To this end, we define respectively $S: U \rightarrow U^{*}$ and $T: U \rightarrow U^{*}$ by

$$
\langle S u, v\rangle=a(u, v), \quad \text { and } \quad\langle T u, v\rangle=-(q u, v), \quad \text { for all } v \in U .
$$


Assumption 4.1(a) implies that $S$ is injective. Further, Lemma 4.2 implies

$$
\begin{aligned}
\|u\|_{U}^{2} \leq c A(u, u) & =c(\langle S u, u\rangle+\langle T u, u\rangle) \\
& \leq c\left(\|S u\|_{U^{*}}+\|T u\|_{U^{*}}\right)\|u\|_{U}, \quad \text { for all } u \in U .
\end{aligned}
$$

Meanwhile, the compactness of $T$ follows from the fact $q \in L^{\infty}(D)$ and the compactness of $U$ in $L^{2}(D)$. Now the Petree-Tartar lemma directly implies that there exists a constant $\gamma>0$ satisfying

$$
\gamma\|u\|_{U} \leq \sup _{v \in U} \frac{a(u, v)}{\|v\|_{U}}
$$

This together with Assumption 4.1 $\left(\mathrm{a}^{*}\right)$ shows that the operator $S: U \rightarrow U^{*}$ is bijective, i.e., there is a unique solution of (4.10) (see, e.g. [13, Corollary A.45]).

We now show that the variational solution $u$ in Theorem 4.3, in fact, is a strong solution when $\langle F, v\rangle=(f, v)$ for some $f \in L^{2}(D)$. We consider the problem

$$
-{ }_{0}^{R} D_{x}^{\alpha} w=f-q u \text {. }
$$

A strong solution is given by (3.3) with a right-hand side $\tilde{f}=f-q u$. It satisfies the variational equation and hence coincides with the unique variational solution. We record this result below.

Theorem 4.4. Let Assumption 4.1 hold, and $q \in L^{\infty}(D)$. Then with a right-hand side $\langle F, v\rangle=(f, v)$ for some $f \in L^{2}(D)$, the solution $u$ to (4.10) is in $\widetilde{H}^{\alpha-1+\beta}(D) \cap$ $\widetilde{H}^{\alpha / 2}(D)$ for any $\beta \in(1-\alpha / 2,1 / 2)$, and it satisfies

$$
\|u\|_{H^{\alpha-1+\beta}} \leq c\|f\|_{L^{2}(D)} .
$$

Proof. It follows from Theorem 4.3 and Assumption 4.1 that there exists a solution $u \in \widetilde{H}^{\alpha / 2}(D)$. Next we rewrite into (4.12) with a right-hand side $\widetilde{f}=f-q u$. In view of the fact that $q \in L^{\infty}(D)$ and $u \in \widetilde{H}^{\alpha / 2}(D)$, there holds $q u \in L^{2}(D)$, and hence $\tilde{f} \in L^{2}(D)$. Now the desired assertion follows directly from the representation (3.3), Theorem 3.1 and Corollary 3.2.

Remark 4.2. In general, the best possible regularity of the solution to (1.1) with a Riemann-Liouville fractional derivative is $H^{\alpha-1+\beta}(D)$ for any $\beta \in(1-\alpha / 2,1 / 2)$, due to the presence of the singular term $x^{\alpha-1}$. The only possibility of an improved regularity is the case $\left({ }_{0} I_{x}^{\alpha} f\right)(1)=0$ (for $\left.q=0\right)$.

We note that a similar result holds for the adjoint problem: given $F \in U^{*}$, find $w \in U$ such that

$$
a(v, w)=\langle v, F\rangle, \quad \text { for all } v \in U .
$$

Then there exists a unique solution $w \in U$ to the adjoint problem. Indeed, Assumption 4.1 and (4.11) immediately imply that the inf-sup condition for the adjoint problem holds with the same constant. Now, by proceeding as in (3.3), for $q=0$ and a right-hand side $\langle F, v\rangle \equiv(f, v)$ with $f \in L^{2}(D)$, we have

$$
w=-{ }_{x} I_{1}^{\alpha} f+\left({ }_{x} I_{1}^{\alpha} f\right)(0)(1-x)^{\alpha-1} .
$$

This implies a similar regularity pickup, i.e., $w \in H^{\alpha-1+\beta}$, for 4.13), provided that $q \in L^{\infty}(D)$. Further, we can repeat the arguments in the proof of Theorem 4.4 for a general $q$ to deduce the regularity of the adjoint solution $w$. We record the observation in a remark. 
Remark 4.3. Let Assumption 4.1 hold, and $q \in L^{\infty}(D)$. Then with a right-hand side $\langle F, v\rangle=(f, v)$ for some $f \in L^{2}(D)$, the solution $w$ to (4.13) is in $H^{\alpha-1+\beta}(D) \cap$ $\widetilde{H}^{\alpha / 2}(D)$ for any $\beta \in(1-\alpha / 2,1 / 2)$, and it satisfies

$$
\|w\|_{H^{\alpha-1+\beta}(D)} \leq c\|f\|_{L^{2}(D)} .
$$

4.3. Variational stability in the Caputo case. We next consider the variational formulation for the Caputo derivative, which, unlike the Riemann-Liouville case, involves a test space $V$ different from the solution space $U$. We set $\phi_{0}=(1-x)^{\alpha-1}$. By Corollary 3.2, $\phi_{0}$ is in the space $\widetilde{H}_{R}^{\alpha / 2}(D)$. Further, we observe that

$$
A\left(u, \phi_{0}\right)=0, \quad \text { for all } u \in U \text {. }
$$

Indeed, for $u \in \widetilde{H}^{1}(D)$, by the change of integration order formula (2.6), there holds

$$
\begin{aligned}
A\left(u,(1-x)^{\alpha-1}\right) & =-\left({ }_{0} I_{x}^{1-\alpha / 2} u^{\prime},{ }_{x}^{R} D_{1}^{\alpha / 2}(1-x)^{\alpha-1}\right) \\
& =c_{\alpha}\left(u^{\prime},{ }_{x} I_{1}^{1-\alpha / 2}(1-x)^{\alpha / 2-1}\right)=c_{\alpha}^{\prime}\left(u^{\prime}, 1\right)=0 .
\end{aligned}
$$

Now for a given $u \in U$, we set $v=u-\gamma_{u} \phi_{0}$, where the linear functional $\gamma_{u}$ is defined by

$$
\gamma_{u}=\frac{\left(x^{1-\alpha}, u\right)}{\left(x^{1-\alpha}, \phi_{0}\right)}
$$

Clearly, the norms $\|\cdot\|_{H^{\alpha / 2}(D)}$ and $\|\cdot\|_{\widetilde{H}_{R}^{\alpha / 2}(D)}$ for $\alpha \in(1,2)$ are equivalent on the space $\widetilde{H}_{R}^{\alpha / 2}(D)$. This together with the continuity of the embedding from $H^{\alpha / 2}(D)$ into $L^{\infty}(D)$ yields

$$
\left|\gamma_{u}\right| \leq c\left|\left(x^{1-\alpha}, u\right)\right| \leq c\|u\|_{L^{\infty}(D)}\left\|x^{1-\alpha}\right\|_{L^{1}(D)} \leq c\|u\|_{\widetilde{H}^{\alpha / 2}(D)} .
$$

Consequently, the function $v$ satisfies $v \in H^{\alpha / 2}(D)$ and $v(1)=0$, i.e., it is in the space $V$. By Lemma 4.2 ,

$$
A(u, v)=A(u, u) \geq c\|u\|_{\widetilde{H}^{\alpha / 2}(D)}^{2} .
$$

Finally, there also holds

$$
\|v\|_{\widetilde{H}_{R}^{\alpha / 2}(D)} \leq\|u\|_{\widetilde{H}^{\alpha / 2}(D)}+c\left|\gamma_{u}\right| \leq c\|u\|_{\widetilde{H}^{\alpha / 2}(D)},
$$

and thus the inf-sup condition follows immediately:

$$
\|u\|_{\widetilde{H}^{\alpha / 2}(D)} \leq c \sup _{v \in V} \frac{A(u, v)}{\|v\|_{\widetilde{H}_{R}^{\alpha / 2}(D)}}, \quad \text { for all } u \in U .
$$

Now given any $v \in V$, we set $u=v-v(0) \phi_{0}$. Obviously, $u$ is nonzero whenever $v \neq 0$ and by applying Lemma 4.2 we get

$$
A(u, v)=A(u, u)>0 .
$$

This implies that if $A(u, v)=0$ for all $u \in U$, then $v=0$. This and (4.16) imply that the corresponding variational problem is stable, i.e., given $F \in V^{*}$, there exists a unique $u \in U$ satisfying

$$
A(u, v)=\langle F, v\rangle, \quad \text { for all } v \in V .
$$

We next consider the case $q \neq 0$ and like before we assume the uniqueness of the bilinear form.

Assumption 4.2. Let the bilinear form $a(u, v)$ with $u \in U, v \in V$ satisfy 
(b) The problem of finding $u \in U$ such that $a(u, v)=0$ for all $v \in V$ has only the trivial solution $u \equiv 0$.

$\left(\mathrm{b}^{*}\right)$ The problem of finding $v \in V$ such that $a(u, v)=0$ for all $u \in U$ has only the trivial solution $v \equiv 0$.

We then have the following existence result.

Theorem 4.5 (Caputo derivative). Let Assumption 4.2 hold and $q \in L^{\infty}(D)$. Then for any given $F \in V^{*}$, there exists a unique solution $u \in U$ to

$$
a(u, v)=\langle F, v\rangle, \quad \text { for all } v \in V .
$$

Proof. The proof is similar to that of Theorem 4.3. In this case, we define $S: U \rightarrow$ $V^{*}$ and $T: U \rightarrow V^{*}$ by

$$
\langle S u, v\rangle=a(u, v) \quad \text { and } \quad\langle T u, v\rangle=-(q u, v), \quad \text { for all } v \in V .
$$

Assumption 4.2(b) implies that $S$ is injective. By applying (4.16) we get for any $u \in U$,

$$
\|u\|_{U} \leq c \sup _{v \in V} \frac{A(u, v)}{\|v\|_{V}} \leq c \sup _{v \in V} \frac{a(u, v)}{\|v\|_{V}}+c \sup _{v \in V} \frac{-(q u, v)}{\|v\|_{V}}=c\left(\|S u\|_{V^{*}}+\|T u\|_{V^{*}}\right) .
$$

The rest of the proof, including verifying the inf-sup condition,

$$
\gamma\|u\|_{U} \leq \sup _{v \in V} \frac{a(u, v)}{\|v\|_{U}}, \quad \text { for all } u \in U
$$

is essentially identical with that of Theorem 4.3 .

To relate the variational solution to the strong solution, we require additional conditions on the potential term $q$ when $\alpha \in(1,3 / 2]$. We first state an "algebraic" property of the space $\widetilde{H}^{s}(D), 0<s \leq 1$, which is reminiscent of [1, Theorem 4.39, pp. 106].

Lemma 4.6. Let $0<s \leq 1, s \neq 1 / 2$. Then for any $u \in \widetilde{H}^{s}(D) \cap L^{\infty}(D)$ and $v \in H^{s}(D) \cap L^{\infty}(D)$, the product $u v$ is in $\widetilde{H}^{s}(D)$.

Proof. Obviously, the product $u v$ has a bounded $L^{2}(D)$-norm, so it suffices to show that the $\widetilde{H}^{s}(D)$-seminorm exists. First we consider the case $s=1$. The trivial identity $(u v)^{\prime}=u^{\prime} v+u v^{\prime}$ yields

$$
\left\|(u v)^{\prime}\right\|_{L^{2}(D)} \leq\|v\|_{L^{\infty}(D)}\left\|u^{\prime}\right\|_{L^{2}(D)}+\|u\|_{L^{\infty}(D)}\left\|v^{\prime}\right\|_{L^{2}(D)}<\infty,
$$

from which the desired assertion follows immediately. For $0<s<1, s \neq 1 / 2$, we use the definition of the $\widetilde{H}^{s}(D)$-seminorm [22]

$$
|v|_{\widetilde{H}^{s}(D)}^{2}=\int_{0}^{1} \int_{0}^{1} \frac{|v(x)-v(y)|^{2}}{|x-y|^{1+2 s}} d x d y, \quad s \neq 1 / 2 .
$$


Using the splitting $u(x) v(x)-u(y) v(y)=(u(x)-u(y)) v(x)+u(y)(v(x)-v(y))$ and the trivial inequality $(a+b)^{2} \leq 2\left(a^{2}+b^{2}\right)$, we deduce that for $s \neq \frac{1}{2}$,

$$
\begin{aligned}
|u v|_{\widetilde{H}^{s}(D)}^{2} & :=\int_{0}^{1} \int_{0}^{1} \frac{(u(x) v(x)-u(y) v(y))^{2}}{(x-y)^{1+2 s}} d x d y \\
\leq & 2 \int_{0}^{1} \int_{0}^{1} \frac{(u(x)-u(y))^{2} v(x)^{2}+u(y)^{2}(v(x)-v(y))^{2}}{(x-y)^{1+2 s}} d x d y \\
\leq & 2\|v\|_{L^{\infty}(D)}^{2} \int_{0}^{1} \int_{0}^{1} \frac{(u(x)-u(y))^{2}}{(x-y)^{1+2 s}} d x d y \\
& +2\|u\|_{L^{\infty}(D)}^{2} \int_{0}^{1} \int_{0}^{1} \frac{(v(x)-v(y))^{2}}{(x-y)^{1+2 s}} d x d y<\infty
\end{aligned}
$$

where in the last line we have used the condition $u \in \widetilde{H}^{s}(D) \cap L^{\infty}(D)$ and $v \in$ $H^{s}(D) \cap L^{\infty}(D)$. Therefore, by the definition of the space $\widetilde{H}^{s}(D)$, we have $u v \in$ $\widetilde{H}^{s}(D)$.

Remark 4.4. The $L^{\infty}(D)$ condition in Lemma 4.6 is only needed for $s \in(0,1 / 2)$, since by Sobolev embedding theorem [1, for $s>1 / 2$, the space $H^{s}(D)$ embeds continuously into $L^{\infty}(D)$. Lemma 4.6 can also be extended to the case $s=1 / 2$ as follows: if $u, v \in L^{\infty}(D) \cap \widetilde{H}^{1 / 2}(D)$, then $u v \in \widetilde{H}^{1 / 2}(D)$. The only change to the proof is that the equivalent norm then reads 22

$$
\|v\|_{\widetilde{H}^{1 / 2}(D)}^{2}=\int_{0}^{1} \int_{0}^{1} \frac{(v(x)-v(y))^{2}}{(x-y)^{2}} d x d y+\int_{0}^{1}\left(\frac{v(x)^{2}}{1-x}+\frac{v(x)^{2}}{x}\right) d x .
$$

Now by introducing $0 \leq \beta<1 / 2$ so that $\alpha+\beta>3 / 2$, then we have the following theorem.

Theorem 4.7. Let $s \in[0,1 / 2)$ and Assumption 4.2 be fulfilled. Suppose that $\langle F, v\rangle=(f, v)$ for some $f \in \widetilde{H}^{s}(D)$ with $\alpha+s>3 / 2$, and $q \in L^{\infty}(D) \cap H^{s}(D)$. Then the variational solution $u \in U$ of (4.17) is in $\widetilde{H}^{\alpha / 2}(D) \cap H^{\alpha+s}(D)$ and it is a solution of (1.1). Further, it satisfies

$$
\|u\|_{H^{\alpha+s}(D)} \leq c\|f\|_{\widetilde{H}^{s}(D)} .
$$

Proof. Let $u$ be the solution of (4.17). We consider the problem

$$
-{ }_{0}^{C} D_{x}^{\alpha} w=f-q u .
$$

By Lemma 4.6, $q u$ is in $\widetilde{H}^{s}(D)$. A strong solution of (4.19) is given by (3.4) with a right-hand side $\tilde{f}=f-q u \in \widetilde{H}^{s}(D)$. Since this solution satisfies the variational problem (4.17), it coincides with $u$. The regularity $u \in H^{\alpha+s}(D)$ is an immediate consequence of Theorem 3.1 .

Remark 4.5. Analogous to the proof of Theorem 4.7, by Theorem 1.4.1.1 of [18, Remark 3.1. and a standard bootstrap argument, one can show that if $f \in \widetilde{H}^{s}(D)$ and $q \in C_{0}^{k}(\bar{D})$ with $k>\lfloor s\rfloor+1$, then the variational solution $u$ is in $H^{s+\alpha}(D)$. We note that the solution to problem (1.1) in the Caputo case can achieve a full regularity, which is in stark contrast with that for the Riemann-Liouville case since for the latter, generally the best possible regularity is $H^{\alpha-1+\beta}(D)$, for any $\beta \in$ $(1-\alpha / 2,1 / 2)$, due to the inherent presence of the singular term $x^{\alpha-1}$. 
Finally, we discuss the adjoint problem in the Caputo case: find $w \in V$ such that

$$
a(v, w)=\langle v, F\rangle, \quad \text { for all } v \in U,
$$

for some fixed $F \in U^{*}$. In the case of $\langle F, v\rangle=(f, v)$ for some $f \in L^{2}(D)$, the strong form reads

$$
-{ }_{x}^{R} D_{1}^{\alpha} w+q w=f,
$$

with $w(1)=0$ and $\left(x^{1-\alpha}, w\right)=0$. By repeating the steps leading to (3.4), we deduce that for $q=0$, the solution $w$ can be expressed as

$$
w=c_{f}(1-x)^{\alpha-1}-{ }_{x} I_{1}^{\alpha} f,
$$

with the prefactor $c_{f}$ given by

$$
c_{f}=\frac{\left(x^{1-\alpha},{ }_{x} I_{1}^{\alpha} f\right)}{\left(x^{1-\alpha},(1-x)^{\alpha-1}\right)}=\frac{\left({ }_{0} I_{x}^{\alpha} x^{1-\alpha}, f\right)}{B(2-\alpha, \alpha)}=\frac{1}{\Gamma(\alpha)}(x, f),
$$

where $B(\cdot, \cdot)$ refers to the Beta function. Clearly, there holds

$$
\left|c_{f}\right| \leq c\left|\left(x^{1-\alpha},{ }_{x} I_{1}^{\alpha} f\right)\right| \leq c\|f\|_{L^{2}(D)} .
$$

Hence $w \in \widetilde{H}^{\alpha / 2}(D) \cap H^{\alpha-1+\beta}(D)$, for any $\beta \in(1-\alpha / 2,1 / 2)$. The case of a general $q$ can be deduced analogously to the proof of Theorem 4.4. Therefore, we have the following improved regularity estimate for the adjoint solution $w$ in the Caputo case.

Theorem 4.8. Let Assumption 4.2 hold, and $q \in L^{\infty}(D)$. Then with a right-hand side $\langle F, v\rangle=(f, v)$ for some $f \in L^{2}(D)$, the solution $w$ to (4.17) is in $\widetilde{H}^{\alpha / 2}(D) \cap$ $H^{\alpha-1+\beta}(D)$ for any $\beta \in(1-\alpha / 2,1 / 2)$, and further there holds

$$
\|w\|_{H^{\alpha-1+\beta}(D)} \leq c\|f\|_{L^{2}(D)} .
$$

Remark 4.6. The adjoint problem for both Caputo and Riemann-Liouville cases is of Riemann-Liouville type, albeit with slightly different boundary conditions, and thus shares the same singularity.

\section{Stability of Finite ElEment approximation}

Now we illustrate the application of the variational formulations developed in Section 4 in the numerical approximation of problem (1.1). We shall analyze the stability of the discrete variational formulations, and derive error estimates for the discrete approximations.

5.1. Finite element spaces and their approximation properties. We introduce a finite element approximation based on an equally spaced partition of the interval $D=(0,1)$. We let $h=1 / m$ be the mesh size with $m>1$ a positive integer, and consider the nodes $x_{i}=i h, i=0, \ldots, m$. We then define $U_{h}$ to be the set of continuous functions in $U$ which are linear when restricted to the subintervals, $\left[x_{i}, x_{i+1}\right], i=0, \ldots, m-1$. Analogously, we define $V_{h}$ to be the set of functions in $V$ which are linear when restricted to the intervals, $\left[x_{i}, x_{i+1}\right], i=0, \ldots, m-1$. Clearly, $U_{h} \subset U$ and $V_{h} \subset V$ implies that functions in either space are continuous and vanish at $x=1$ and functions in $U_{h}$ vanish at $x=0$ while $v_{h} \in V_{h}$ satisfies the integral constraint $\left(x^{1-\alpha}, v_{h}\right)=0$. $V_{h}$.

We first show the approximation properties of the finite element spaces $U_{h}$ and 
Lemma 5.1. Let the mesh $\mathcal{T}_{h}$ be quasi-uniform, and $\alpha / 2 \leq \gamma \leq 2$. If $u \in H^{\gamma}(D) \cap$ $\widetilde{H}^{\alpha / 2}(D)$, then

$$
\inf _{v \in U_{h}}\|u-v\|_{H^{\alpha / 2}(D)} \leq c h^{\gamma-\alpha / 2}\|u\|_{H^{\gamma}(D)} .
$$

Further, if $u \in H^{\gamma}(D) \cap V$, then

$$
\inf _{v \in V_{h}}\|u-v\|_{H^{\alpha / 2}(D)} \leq c h^{\gamma-\alpha / 2}\|u\|_{H^{\gamma}(D)} .
$$

Proof. Let $\Pi_{h} u \in U_{h}$ be the standard Lagrange finite element interpolant of $u$ so that for any $0 \leq s \leq 1$, $\inf _{v \in U_{h}}\|u-v\|_{H^{s}(D)} \leq\left\|u-\Pi_{h} u\right\|_{H^{s}(D)}$. Then the estimate (5.1) is an immediate consequence of the local approximation properties of the interpolant $\Pi_{h} u$ [13, Corollary 1.109, pp. 61] for $p=2$ and Sobolev spaces of integer order. The result for the intermediate fractional values follows from interpolation.

Now we study the approximation properties of the finite element space $V_{h}$. Given a function $u \in V$ we approximate it by a finite element function $\chi$ with $\chi(1)=0$, e.g., the interpolation function, so that $\|u-\chi\|_{H^{\alpha / 2}(D)} \leq c h^{\gamma-\alpha / 2}\|u\|_{H^{\gamma}(D)}$. Then we introduce the projection operator $P: H^{\alpha / 2}(D) \rightarrow V$ by $P u=u-\gamma_{u}(1-x)$ where $\gamma_{u}=\left(u, x^{1-\alpha}\right) /\left(x^{1-\alpha}, 1-x\right)$ ensures that $P u \in V$. Now in view of the fact that $P \chi \in V_{h}$ and $P$ is bounded on $H^{\alpha / 2}(D)$-norm, for $u \in V$, we get

$$
\|u-P \chi\|_{H^{\alpha / 2}(D)}=\|P(u-\chi)\|_{H^{\alpha / 2}(D)} \leq c\|u-\chi\|_{H^{\alpha / 2}(D)},
$$

and this completes the proof.

5.2. Error estimates in the Riemann-Liouville case. In this case the finite element source problem is to find $u_{h} \in U_{h}$ such that

$$
a\left(u_{h}, v\right)=\langle F, v\rangle, \quad \text { for all } v \in U_{h} .
$$

Here $F \in U^{*}$ is a bounded linear functional on the space $U$. In Theorem 5.3 below we establish error estimates on the finite element approximation $u_{h}$ when the source term is given by $\langle F, v\rangle \equiv(f, v)$ with $f \in L^{2}(D)$. To derive the error estimates, we first establish a stability result for the discrete variational formulation, using a technique analogous to that of Schatz [29, from which the existence and uniqueness of a discrete solution $u_{h}$ follows directly.

Lemma 5.2. Let Assumption 4.1 hold, $f \in L^{2}(D)$, and $q \in L^{\infty}(D)$. Then there exists an $h_{0}$ such that for all $h \leq h_{0}$ and a constant $c>0$ satisfying

$$
c\left\|z_{h}\right\|_{U} \leq \sup _{v \in U_{h}} \frac{a\left(z_{h}, v\right)}{\|v\|_{U}}, \quad \text { for all } z_{h} \in U_{h} .
$$

For such $h$, the finite element problem: Find $u_{h} \in U_{h}$ such that

$$
a\left(u_{h}, v\right)=(f, v), \quad \text { for all } v \in U_{h},
$$

has a unique solution.

Proof. The existence and uniqueness of the discrete solution $u_{h}$ when $q=0$ is an immediate consequence of Lemma 4.2. The existence and uniqueness of the solutions to the more general $(q \neq 0)$ discrete problem is equivalent to the invertibility of the stiffness matrix. Since the stiffness matrix is square, its invertibility follows from uniqueness and is a simple consequence of (5.4). 
To prove (5.4), we first define $R_{h}: U \rightarrow U_{h}$ defined by $A\left(v, R_{h} u\right)=A(v, u)$ for all $v \in U_{h}$. Céa's lemma and finite element duality imply that this problem is uniquely solvable with the solution $R_{h} u$ satisfying

$$
\begin{aligned}
\left\|R_{h} u\right\|_{H^{\alpha / 2}(D)} & \leq c\|u\|_{H^{\alpha / 2}(D)}, \\
\left\|u-R_{h} u\right\|_{L^{2}(D)} & \leq c h^{\alpha / 2-1+\beta}\|u\|_{H^{\alpha / 2}(D)},
\end{aligned}
$$

for $\beta \in(1-\alpha / 2,1 / 2)$. We next use a kick-back argument. For any $z_{h} \in U_{h} \subset U$ due to the inf-sup condition (4.11) we have

$$
\gamma\left\|z_{h}\right\|_{U} \leq \sup _{v \in U} \frac{a\left(z_{h}, v\right)}{\|v\|_{U}} \leq \sup _{v \in U} \frac{a\left(z_{h}, v-R_{h} v\right)}{\|v\|_{U}}+\sup _{v \in U} \frac{a\left(z_{h}, R_{h} v\right)}{\|v\|_{U}} .
$$

Now using the fact that $q \in L^{\infty}(D)$, the error estimate in (5.6), and the regularity pickup with $\beta \in(1-\alpha / 2,1 / 2)$ we get the following bound for the first term

$$
\begin{aligned}
\sup _{v \in U} \frac{a\left(z_{h}, v-R_{h} v\right)}{\|v\|_{U}} & =\sup _{v \in U} \frac{\left(q z_{h}, v-R_{h} v\right)}{\|v\|_{U}} \\
& \leq \operatorname{csup}_{v \in U} \frac{\left\|z_{h}\right\|_{L^{2}(D)}\left\|v-R_{h} v\right\|_{L^{2}(D)}}{\|v\|_{U}} \leq c h^{\alpha / 2-1+\beta}\left\|z_{h}\right\|_{U} .
\end{aligned}
$$

For the second term we use the stability estimate in (5.6) for the operator $R_{h}$ to get

$$
\sup _{v \in U} \frac{a\left(z_{h}, R_{h} v\right)}{\|v\|_{U}} \leq c \sup _{v \in U} \frac{a\left(z_{h}, R_{h} v\right)}{\left\|R_{h} v\right\|_{U}}=c \sup _{v \in U_{h}} \frac{a\left(z_{h}, v\right)}{\|v\|_{U}} .
$$

Now with the choice $h_{0}^{\alpha / 2-1+\beta}=\gamma /(2 c)$ we arrive at the discrete inf-sup condition for all $h<h_{0}$.

Now we give the error estimate for the discrete approximation $u_{h}$ in the RiemannLiouville case.

Theorem 5.3. Let Assumption 4.1 hold, $f \in L^{2}(D)$, and $q \in L^{\infty}(D)$. Then there is an $h_{0}$ such that for all $h \leq h_{0}$, the solution $u_{h}$ to the finite element problem (5.5) satisfies, for any $\beta \in(1-\alpha / 2,1 / 2)$,

$$
\left\|u-u_{h}\right\|_{L^{2}(D)}+h^{\alpha / 2-1+\beta}\left\|u-u_{h}\right\|_{H^{\alpha / 2}(D)} \leq c h^{\alpha-2+2 \beta}\|f\|_{L^{2}(D)} .
$$

Proof. The proof is standard given Lemma 5.2. Lemma 5.1, and Theorem 4.4.

Remark 5.1. In view of classical interpolation error estimates and the stability estimate in Theorem 4.4 the $H^{\alpha / 2}(D)$-norm estimate of the error for the FEM approximation $u_{h}$ is optimal, whereas the $L^{2}(D)$-norm error estimate is suboptimal, which is also confirmed by numerical experiments. The culprit of the suboptimality is due to the limited global regularity of the adjoint solution; cf. Remark 4.3.

Remark 5.2. The analysis for the adjoint problem (4.13) is similar. The discrete inf-sup condition for the adjoint problem holds (with the same constant) whenever (5.4) holds. Accordingly, the discrete adjoint problem: find $w_{h} \in U_{h}$ such that

$$
a\left(v, w_{h}\right)=(v, f), \quad \text { for all } v \in U_{h},
$$

for a given right-hand side $f \in L^{2}(D)$ is uniquely solvable when $h<h_{0}$ and satisfies for any $\beta \in(1-\alpha / 2,1 / 2)$,

$$
\left\|w-w_{h}\right\|_{L^{2}(D)}+h^{\alpha / 2-1+\beta}\left\|w-w_{h}\right\|_{H^{\alpha / 2}(D)} \leq c h^{\alpha-2+2 \beta}\|f\|_{L^{2}(D)} .
$$


5.3. Error estimates in the Caputo case. In the case of the Caputo derivative, the finite element source problem is to find $u_{h} \in U_{h}$ satisfying

$$
a\left(u_{h}, v\right)=\langle F, v\rangle, \quad \text { for all } v \in V_{h} .
$$

Here $F \in V^{*}$ is a bounded linear functional on the space $V$. The existence and uniqueness of the discrete solution $u_{h}$ even when $q=0$ is not as immediate as the Riemann-Liouville case. The discrete inf-sup condition in this "simplest" case $q=0$ is stated in the lemma below.

Lemma 5.4. There is an $h_{0}>0$ and $c$ independent of $h$ such that for all $h \leq h_{0}$ and $w_{h} \in U_{h}$,

$$
\left\|w_{h}\right\|_{U} \leq c \sup _{v_{h} \in V_{h}} \frac{A\left(w_{h}, v_{h}\right)}{\left\|v_{h}\right\|_{V}}
$$

Proof. Let $w_{h}$ be in $U_{h}$ and set $v=w_{h}-\gamma_{w_{h}} \phi_{0}\left(\right.$ recall $\left.\phi_{0}=(1-x)^{\alpha-1} \in \widetilde{H}_{R}^{\alpha / 2}(D)\right)$. The argument leading to (4.16) implies $A\left(w_{h}, v\right) \geq c\left\|w_{h}\right\|_{\widetilde{H}^{\alpha / 2}(D)}^{2}$ and consequently, there holds

$$
\|v\|_{\widetilde{H}_{R}^{\alpha / 2}(D)} \leq c\left\|w_{h}\right\|_{\widetilde{H}^{\alpha / 2}(D)} \cdot
$$

However, since $\phi_{0}$ is not piecewise linear, $v \notin V_{h}$. Next we set $\widetilde{v}_{h}=\Pi_{h} v=$ $w_{h}-\gamma_{w_{h}} \Pi_{h} \phi_{0}$, where $\Pi_{h}$ denotes the Lagrangian interpolation operator. We clearly have

$$
\begin{aligned}
\left\|v-\widetilde{v}_{h}\right\|_{H^{\alpha / 2}(D)} & =\left|\gamma_{w_{h}}\right|\left\|\left(I-\Pi_{h}\right) \phi_{0}\right\|_{H^{\alpha / 2}(D)} \\
& \leq c\left|\gamma_{w_{h}}\right| h^{\alpha / 2-1+\beta}\left\|\phi_{0}\right\|_{\widetilde{H}_{R}^{\alpha-1+\beta}(D)}
\end{aligned}
$$

for any $\beta \in(1-\alpha / 2,1 / 2)$ by Lemma 3.2 Now in order to obtain a function $v_{h}$ in the space $V_{h}$, we apply the projection operator $P: \widetilde{H}^{\alpha / 2}(D) \rightarrow V$ defined in the proof of Lemma 5.1 to $\widetilde{v}_{h}$, and set $v_{h}=P \widetilde{v}_{h}$. By the stability of the operator $P$ in $H^{\alpha / 2}(D)$ and using $\left|\gamma_{w_{h}}\right| \leq c\left\|w_{h}\right\|_{U}$, we deduce

$$
\left\|v-v_{h}\right\|_{H^{\alpha / 2}(D)}=\left\|P\left(v-\widetilde{v}_{h}\right)\right\|_{H^{\alpha / 2}(D)} \leq c h^{\alpha / 2-1+\beta}\left\|w_{h}\right\|_{U} .
$$

This together with (5.8) and the triangle inequality also implies that $\left\|v_{h}\right\|_{H^{\alpha / 2}(D)} \leq$ $c\left\|w_{h}\right\|_{U}$. Thus,

$$
\begin{aligned}
\left\|w_{h}\right\|_{U} \leq \frac{c A\left(w_{h}, v\right)}{\left\|w_{h}\right\|_{U}} & \leq \frac{c A\left(w_{h}, v_{h}\right)}{\left\|w_{h}\right\|_{U}}+\frac{c A\left(w_{h}, v-v_{h}\right)}{\left\|w_{h}\right\|_{U}} \\
& \leq \frac{c A\left(w_{h}, v_{h}\right)}{\left\|w_{h}\right\|_{U}}+c_{1} h^{\alpha / 2-1+\beta}\left\|w_{h}\right\|_{U} .
\end{aligned}
$$

By taking $h_{0}$ so that $c_{1} h_{0}^{\alpha / 2-1+\beta} \leq 1 / 2$ we get

$$
\left\|w_{h}\right\|_{U} \leq \frac{c A\left(w_{h}, v_{h}\right)}{\left\|w_{h}\right\|_{U}} \leq \frac{c A\left(w_{h}, v_{h}\right)}{\left\|v_{h}\right\|_{V}}
$$

from which the lemma immediately follows.

Now we can show the inf-sup condition for the bilinear form $a(\cdot, \cdot)$ with a general $q$ defined on $U_{h} \times V_{h}$.

Lemma 5.5. Let Assumption 4.2 be fulfilled. Then there is an $h_{0}>0$ such that for all $h \leq h_{0}$,

$$
\left\|w_{h}\right\|_{U} \leq c \sup _{v_{h} \in V_{h}} \frac{a\left(w_{h}, v_{h}\right)}{\left\|v_{h}\right\|_{V}}, \quad \text { for all } w_{h} \in U_{h}
$$


Proof. We define $R_{h}: V \rightarrow V_{h}$ by $A\left(u, R_{h} v\right)=A(u, v)$ for all $u \in U_{h}$. That $R_{h}$ satisfies (5.6) follows easily from the adjoint analogue of Lemma 5.4. The remainder of proof is similar to that for Lemma 5.2 but replacing (4.11) with (4.18).

Finally we state an error estimate on the finite element approximation $u_{h}$ when the source term is given by $\langle F, v\rangle \equiv(f, v)$ with $f \in \widetilde{H}^{s}(D)$ with $s \in[0,1 / 2)$ such that $\alpha+s>3 / 2$.

Theorem 5.6. Let Assumption 4.2 hold, $f \in \widetilde{H}^{s}(D)$, and $q \in L^{\infty}(D) \cap \widetilde{H}^{s}(D)$ for some $s \in[0,1 / 2)$ such that $\alpha+s>3 / 2$. Then there is an $h_{0}$ such that for $h \leq h_{0}$, the finite element problem: Find $u_{h} \in U_{h}$ satisfying

$$
a\left(u_{h}, v\right)=(f, v), \quad \text { for all } v \in V_{h},
$$

has a unique solution. Moreover, the solution $u_{h}$ satisfies that, for any $\beta \in[\alpha / 2-$ $1 / 2,1 / 2)$,

$$
\begin{aligned}
\left\|u-u_{h}\right\|_{\widetilde{H}^{\alpha / 2}(D)} & \leq c h^{\min (\alpha+s, 2)-\alpha / 2}\|f\|_{\widetilde{H}^{s}(D)}, \\
\left\|u-u_{h}\right\|_{L^{2}(D)} & \leq c h^{\min (\alpha+s, 2)-1+\beta}\|f\|_{\widetilde{H}^{s}(D)} .
\end{aligned}
$$

Proof. According to Theorem 4.7 the solution $u$ to (5.9) is in $H^{\alpha+s}(D) \cap \widetilde{H}^{\alpha / 2}(D)$. By Lemma 5.5, the discrete solution $u_{h}$ is well defined. The $\widetilde{H}^{\alpha / 2}(D)$-norm estimate of the error $e=u_{h}-u$ follows from Céa's lemma (cf. e.g. [13, pp. 96, Lemma 2.28]). To derive the $L^{2}(D)$ estimate, we appeal again to the Nitsche's trick and introduce the adjoint problem: find $w \in V$ such that

$$
a(v, w)=(v, e), \quad \text { for all } v \in U .
$$

By Theorem 4.8, the solution $w$ lies in the space $\widetilde{H}_{R}^{\alpha-1+\beta}(D)$ for any $\beta \in[\alpha / 2-$ $1 / 2,1 / 2)$, and satisfies the a priori estimate $\|w\|_{H^{\alpha-1+\beta}(D)} \leq c\|e\|_{L^{2}(D)}$. The rest of the proof is identical with that of Theorem 5.3 .

Remark 5.3. Like in the case of Riemann-Liouville fractional derivative, the $L^{2}(D)$ norm of the error is suboptimal due to the insufficient regularity of the adjoint solution; cf. Theorem 4.8 .

\section{Fractional Sturm-Liouville Problem}

In this section we apply the variational formulation in Section 4 to the FSLP (1.2). The weak formulation of the eigenvalue problem (1.2) reads: find $u \in U$ and $\lambda \in \mathbb{C}$ such that

$$
a(u, v)=\lambda(u, v), \quad \text { for all } v \in V .
$$

Accordingly, the discrete problem is given by: find an approximation $u_{h} \in U_{h}$ and $\lambda_{h} \in \mathbb{C}$ such that

$$
a\left(u_{h}, v\right)=\lambda_{h}\left(u_{h}, v\right), \quad \text { for all } v \in V_{h} .
$$

Following [3, we introduce the operator $T: L^{2}(D) \rightarrow \widetilde{H}^{\alpha / 2}(D)$ defined by

$$
T f \in \widetilde{H}^{\alpha / 2}(D), \quad a(T f, v)=(f, v), \quad \text { for all } v \in V .
$$

Obviously, $T$ is the solution operator of the source problem (1.1). According to Theorems 4.4 and 4.7, the solution operator $T$ satisfies the following smoothing property:

$$
\|T f\|_{H^{\alpha / 2}(D)} \leq c\|f\|_{L^{2}(D)}
$$


Since the space $\widetilde{H}^{\alpha / 2}(D)$ is compactly embedded into $L^{2}(D)$, we deduce that $T: L^{2}(D) \rightarrow L^{2}(D)$ is a compact operator. Meanwhile, by viewing $T$ as an operator on the space $H^{\alpha / 2}(D)$ and using the regularity pickup established in Theorems 4.4 and 4.7 we can show that $T: H^{\alpha / 2}(D) \rightarrow H^{\alpha / 2}(D)$ is compact. Then it follows immediately from (6.3) that $(\lambda, u)$ is an eigenpair of (6.1) if and only if

$$
T u=\lambda^{-1} u, \quad u \neq 0,
$$

i.e., if and only if $(\mu=1 / \lambda, u)$ is an eigenpair of the operator $T$. With the help of this correspondence, the properties of the eigenvalue problem (6.1) can be derived from the spectral theory for compact operators [11. Let $\sigma(T) \subset \mathbb{C}$ be the set of all eigenvalues of $T$ (or its spectrum), which is known to be a countable set with no nonzero limit points. Due to Assumptions 4.1 and 4.2 on the bilinear form $a(u, v)$, zero is not an eigenvalue of $T$. Furthermore, for any $\mu \in \sigma(T)$, the space $N(\mu I-T)$, where $N$ denotes the null space, of eigenvectors corresponding to $\mu$ is finite dimensional.

Now let $T_{h}: U_{h} \rightarrow U_{h}$ be a family of operators for $0<h<1$ defined by

$$
T_{h} f \in U_{h}, \quad a\left(T_{h} f, v\right)=(f, v), \quad \text { for all } v \in V_{h} .
$$

Next we apply the abstract approximation theory for the spectrum of variationally formulated eigenvalue problems [3, Section 8]. To this end, we need to establish the approximation properties of (6.2). Let $\lambda^{-1} \in \sigma(T)$ be an eigenvalue of $T$ with algebraic multiplicity $m$. Since $T_{h} \rightarrow T$ in norm, $m$ eigenvalues $\lambda_{h}^{1}, \ldots, \lambda_{h}^{m}$ of $T_{h}$ will converge to $\lambda$. The eigenvalues $\lambda_{h}^{j}$ are counted according to the algebraic multiplicity of $\mu_{h}^{j}=1 / \lambda_{h}^{j}$ as eigenvalues of $T_{h}$. Further, we define the following finite dimensional spaces:

$$
\begin{gathered}
M(\lambda)=\left\{u:\|u\|_{H^{\alpha / 2}(D)}=1\right. \text { a generalized eigenvector } \\
\text { of (16.1) corresponding to } \lambda\}, \\
M^{*}(\lambda)=\left\{u:\|u\|_{H^{\alpha / 2}(D)}=1\right. \text { a generalized adjoint eigenvector } \\
\text { of (6.1) corresponding to } \lambda\},
\end{gathered}
$$

and the following quantities:

$$
\begin{aligned}
& \epsilon_{h}=\epsilon_{h}(\lambda)=\sup _{u \in M(\lambda)} \inf _{v \in U_{h}}\|u-v\|_{H^{\alpha / 2}(D)}, \\
& \epsilon_{h}^{*}=\epsilon_{h}^{*}(\lambda)=\sup _{u \in M^{*}(\lambda)} \inf _{v \in V_{h}}\|u-v\|_{H^{\alpha / 2}(D)} .
\end{aligned}
$$

Then we have the following estimates on $\epsilon_{h}$ and $\epsilon_{h}^{*}$.

Lemma 6.1. Let $r \in(0, \alpha / 2-1 / 2)$. Then the following error bounds for $\epsilon_{h}$ and $\epsilon_{h}^{*}$ are valid.

(a) For Caputo derivative: if Assumption 4.2 holds, $q \in \widetilde{H}^{s}(D) \cap L^{\infty}(D)$, $0 \leq s \leq 1$, such that $\alpha+s>3 / 2$, then $\epsilon_{h} \leq \operatorname{ch}^{\min (\alpha+s, 2)-\alpha / 2}$ and $\epsilon_{h}^{*} \leq C h^{r}$.

(b) For Riemann-Liouville derivative: if Assumption 4.1 holds, then $\epsilon_{h} \leq \mathrm{ch}^{r}$ and $\epsilon_{h}^{*} \leq C h^{r}$.

Proof. The needed regularity for the Caputo case is a simple consequence of Theorem 4.7. We consider the only the case $q \in \widetilde{H}^{s}(D) \cap L^{\infty}(D)$. Since the right-hand side of the problem (1.2) is $\lambda u \in \widetilde{H}^{\alpha / 2}(D)$, we have $u \in \widetilde{H}^{\alpha / 2}(D) \cap H^{\alpha+s}(D)$ in 
view of Theorem 4.7 Next let $\Pi_{h} u \in U_{h}$ be the finite element interpolant of $u$. Then by Lemma 5.1 we deduce that

$$
\left\|u-\Pi_{h} u\right\|_{\widetilde{H}^{\gamma}(D)} \leq h^{\min (\alpha+s, 2)-\gamma}\|u\|_{H^{\min (\alpha+s, 2)}(D)}
$$

for any $0 \leq \gamma \leq 1$. Therefore, by $\|u\|_{H^{\alpha+s}(D)} \leq c$, we get

$$
\epsilon_{h}=\sup _{u \in M(\lambda)} \inf _{v \in U_{h}}\|u-v\|_{H^{\alpha / 2}(D)} \leq \sup _{u \in M(\lambda)}\left\|u-\Pi_{h} u\right\|_{H^{\alpha / 2}(D)} \leq \operatorname{ch}^{\min (\alpha+s, 2)-\alpha / 2} .
$$

The estimate $\epsilon_{h}^{*}$ follows from Theorem 4.8 The Riemann-Liouville case follows analogously from regularity estimates for the source problem and adjoint source problem in Theorem 4.4 and Remark 4.2, respectively.

Now we state the main result for the approximation error of the eigenvalues of problem (1.2). It follows immediately from [3. Theorem 8.3 and pp. 683-714] and Lemma 6.1.

Theorem 6.2. For $\mu \in \sigma(T)$, let $\delta$ be the smallest integer $k$ such that $N((\mu-$ $\left.T)^{k}\right)=N\left((\mu-T)^{k+1}\right)$. Suppose that for each $h$ there is a unit vector $w_{h}^{j}$ satisfying $\left(\left(\lambda_{h}^{j}\right)^{-1}-T_{h}\right)^{k} w_{h}^{j}=0, j=1, \ldots, m$ for some integer $k \leq \delta$.

(a) For Caputo derivative, if Assumption 4.2 holds, $q \in \widetilde{H}^{s}(D) \cap L^{\infty}(D)$ for some $0 \leq s \leq 1$ and $\alpha+s>3 / 2$, then for any $\gamma<\min (\alpha+s, 2)-1 / 2$, there holds $\left|\lambda-\lambda_{h}^{j}\right| \leq \operatorname{ch}^{\gamma / \delta}$.

(b) For Riemann-Liouville derivative, if Assumption 4.1 holds, and $q \in L^{\infty}(D)$, then for any $\gamma<\alpha-1$, there holds $\left|\lambda-\lambda_{h}^{j}\right| \leq \operatorname{ch}^{\gamma / \delta}$.

Remark 6.1. In the case of a zero potential $q \equiv 0$, it is known that the eigenvalues $\lambda$ are zeros of the Mittag-Leffler functions $E_{\alpha, \alpha}(-\lambda)$ and $E_{\alpha, 2}(-\lambda)$ (cf. (7.1) below), for the Riemann-Liouville and Caputo case, respectively. This connection implies that all eigenvalues are simple [27, Section 4.4], and hence the multiplicity $\delta=1$. In our computations we observed that for all potentials $q$ the eigenvalues are simple.

Remark 6.2. The (theoretical) rate of convergence for the Riemann-Liouville case is lower than that for the Caputo case. This is due to limited smoothing property for the Riemann-Liouville derivative operator. It naturally suggests that an adaptive procedure involving a proper grid refinement or enriching the solution space should be used. Nonetheless, a second-order convergence is observed for the eigenvalue approximations, i.e., the abstract theory gives only suboptimal convergence rates.

\section{Numerical EXPERIMENTS AND DISCUSSIONS}

In this section we present some numerical experiments to illustrate the theory, i.e., the sharpness of the finite element error estimates, and the difference between the regularity estimates for the Riemann-Liouville and Caputo derivatives.

7.1. Source problem. We consider the following three examples:

(a) The source term $f=x(1-x)$ belongs to $\widetilde{H}^{s}(D)$ for any $s \in[1,3 / 2)$.

(b) The source term $f=1$ belongs to $\widetilde{H}^{s}(D)$ for any $s \in[0,1 / 2)$.

(c) The source term $f=x^{-1 / 4}$ belongs to the space $\widetilde{H}^{s}(D)$ for any $s \in[0,1 / 4)$.

The computations were performed on uniform meshes of mesh sizes $h=1 /\left(2^{k} \times\right.$ $10), k=1,2, \ldots, 7$. In the examples, we set the potential term $q$ to zero, so that the exact solution can be computed directly using the solution representations (3.3) 
and (3.4). For each example, we consider three different $\alpha$ values, i.e., $7 / 4,3 / 2$ and $4 / 3$, and present the $L^{2}(D)$ - and $H^{\alpha / 2}(D)$-norm of the error $e=u-u_{h}$.

7.1.1. Numerical results for example (a). The source term $f$ is smooth, and the true solution $u$ is given by

$u(x)=\left\{\begin{array}{cl}\frac{1}{\Gamma(\alpha+2)}\left(x^{\alpha-1}-x^{\alpha+1}\right)-\frac{2}{\Gamma(\alpha+3)}\left(x^{\alpha-1}-x^{\alpha+2}\right), & \text { Riemann-Liouville case, } \\ \frac{1}{\Gamma(\alpha+2)}\left(x-x^{\alpha+1}\right)-\frac{2}{\Gamma(\alpha+3)}\left(x-x^{\alpha+2}\right), & \text { Caputo case. }\end{array}\right.$

According to Theorems 4.4 and 4.7 the solution $u$ belongs to $\widetilde{H}_{L}^{\alpha-1+\beta}(D)$ and $H^{\alpha+1+\beta}(D)$ for any $\beta \in(1-\alpha / 2,1 / 2)$ for the Riemann-Liouville and Caputo case, respectively. In particular, in the Caputo case, the solution $u$ belongs to $H^{2}(D)$. Hence, in the Riemann-Liouville case, the finite element approximations $u_{h}$ would converge at a rate $O\left(h^{\alpha / 2-1 / 2}\right)$ and $O\left(h^{\alpha-1}\right)$ in $H^{\alpha / 2}(D)$-norm and $L^{2}(D)$-norm, respectively, whereas that for the Caputo case would be at a rate $O\left(h^{2-\alpha / 2}\right)$ and $O\left(h^{3 / 2}\right)$, respectively. We note that despite the good regularity of the source term $f$, the Riemann-Liouville solution is nonsmooth, due to the presence of the term $x^{\alpha-1}$, and hence the numerical scheme can only converge slowly in the $H^{\alpha / 2}(D)$ norm. The numerical results are shown in Tables 1 and 2 , where the number in the bracket under the column rate is the theoretical convergence rate. The results indicate that the $H^{\alpha / 2}(D)$ estimates are fully confirmed; however, the $L^{2}(D)$ estimates are suboptimal. The actual convergence rate in $L^{2}(D)$-norm is one half order higher than the theoretical one, for both fractional derivatives, which agrees with Remark 5.1. Intuitively, this might be explained by the structure of the adjoint problem: The adjoint solution contains the singular term $(1-x)^{\alpha-1}$, with a coefficient $\left({ }_{x} I_{1}^{\alpha} e\right)(0)=\frac{1}{\Gamma(\alpha)} \int_{0}^{1} x^{\alpha-1} e(x) d x$ for the Riemann-Liouville case (respectively $\frac{1}{\Gamma(\alpha)}(x, e)$ for the Caputo case); the error function $e$ has large oscillations mainly around the origin, which is however compensated by the weight $x^{\alpha-1}$ in the Riemann-Liouville case (the weight is $x$ in the Caputo case) in the integral, and thus the coefficient is much smaller than the apparent $L^{2}(D)$-norm of the error. This can be numerically confirmed: the coefficient tends much faster to zero than the $L^{2}(D)$-norm of the error (cf. Table 3), and hence in the adjoint technique for deriving the $L^{2}$-error estimate, the term $x^{\alpha-1}$ plays a less significant role than that in the primal problem.

TABLE 1. Numerical results for example (a) with a RiemannLiouville fractional derivative, $f=x(1-x)$, mesh size $h=1 /\left(2^{k} \times\right.$ $10)$.

\begin{tabular}{|c|c|c|c|c|c|c|c|c|c|}
\hline$\alpha$ & $k$ & 1 & 2 & 3 & 4 & 5 & 6 & 7 & rate \\
\hline \multirow{3}{*}{$7 / 4$} & $L^{2}$-norm & $1.70 \mathrm{e}-4$ & $7.05 \mathrm{e}-5$ & $2.95 \mathrm{e}-5$ & $1.24 \mathrm{e}-5$ & $5.21 \mathrm{e}-6$ & $2.19 \mathrm{e}-6$ & $9.21 \mathrm{e}-7$ & $1.25(0.75)$ \\
\cline { 2 - 9 } & $H^{\alpha / 2}$-norm & $8.53 \mathrm{e}-3$ & $6.43 \mathrm{e}-3$ & $4.91 \mathrm{e}-3$ & $3.77 \mathrm{e}-3$ & $2.90 \mathrm{e}-3$ & $2.23 \mathrm{e}-3$ & $1.72 \mathrm{e}-3$ & $0.39(0.38)$ \\
\hline \multirow{3}{*}{$3 / 2$} & $L^{2}$-norm & $1.08 \mathrm{e}-3$ & $5.40 \mathrm{e}-4$ & $2.70 \mathrm{e}-4$ & $1.35 \mathrm{e}-4$ & $6.74 \mathrm{e}-5$ & $3.37 \mathrm{e}-5$ & $1.68 \mathrm{e}-5$ & $1.00(0.50)$ \\
\cline { 2 - 9 } & $H^{\alpha / 2}$-norm & $2.85 \mathrm{e}-2$ & $2.39 \mathrm{e}-2$ & $2.00 \mathrm{e}-2$ & $1.68 \mathrm{e}-2$ & $1.41 \mathrm{e}-2$ & $1.18 \mathrm{e}-2$ & $9.82 \mathrm{e}-3$ & $0.26(0.25)$ \\
\hline \multirow{2}{*}{$4 / 3$} & $L^{2}$-norm & $3.50 \mathrm{e}-3$ & $1.96 \mathrm{e}-3$ & $1.10 \mathrm{e}-3$ & $6.16 \mathrm{e}-4$ & $3.46 \mathrm{e}-4$ & $1.94 \mathrm{e}-4$ & $1.09 \mathrm{e}-4$ & $0.83(0.33)$ \\
\cline { 2 - 9 } & $H^{\alpha / 2}$-norm & $5.40 \mathrm{e}-2$ & $4.79 \mathrm{e}-2$ & $4.25 \mathrm{e}-2$ & $3.76 \mathrm{e}-2$ & $3.33 \mathrm{e}-2$ & $2.93 \mathrm{e}-2$ & $2.58 \mathrm{e}-2$ & $0.18(0.17)$ \\
\hline
\end{tabular}


TABLE 2. Numerical results for example (a) with a Caputo fractional derivative, $f=x(1-x)$, mesh size $h=1 /\left(2^{k} \times 10\right)$.

\begin{tabular}{|c|c|c|c|c|c|c|c|c|c|}
\hline$\alpha$ & $k$ & 1 & 2 & 3 & 4 & 5 & 6 & 7 & rate \\
\hline \multirow{3}{*}{$7 / 4$} & $L^{2}$-norm & $2.45 \mathrm{e}-5$ & $5.98 \mathrm{e}-6$ & $1.48 \mathrm{e}-6$ & $3.72 \mathrm{e}-7$ & $9.38 \mathrm{e}-8$ & $2.37 \mathrm{e}-8$ & $6.00 \mathrm{e}-9$ & $2.00(1.50)$ \\
\cline { 2 - 10 } & $H^{\alpha / 2}$-norm & $1.50 \mathrm{e}-3$ & $6.88 \mathrm{e}-4$ & $3.15 \mathrm{e}-4$ & $1.44 \mathrm{e}-4$ & $6.62 \mathrm{e}-5$ & $3.04 \mathrm{e}-5$ & $1.39 \mathrm{e}-5$ & $1.13(1.13)$ \\
\hline \multirow{3}{*}{$3 / 2$} & $L^{2}$-norm & $4.93 \mathrm{e}-5$ & $1.25 \mathrm{e}-5$ & $3.14 \mathrm{e}-6$ & $7.92 \mathrm{e}-7$ & $1.99 \mathrm{e}-7$ & $4.99 \mathrm{e}-8$ & $1.25 \mathrm{e}-8$ & $1.99(1.50)$ \\
\cline { 2 - 10 } & $H^{\alpha / 2}$-norm & $8.84 \mathrm{e}-4$ & $3.69 \mathrm{e}-4$ & $1.54 \mathrm{e}-4$ & $6.48 \mathrm{e}-5$ & $2.72 \mathrm{e}-5$ & $1.14 \mathrm{e}-5$ & $4.81 \mathrm{e}-6$ & $1.25(1.25)$ \\
\hline \multirow{2}{*}{$4 / 3$} & $L^{2}$-norm & $7.40 \mathrm{e}-5$ & $1.85 \mathrm{e}-5$ & $4.62 \mathrm{e}-6$ & $1.16 \mathrm{e}-6$ & $2.89 \mathrm{e}-7$ & $7.24 \mathrm{e}-8$ & $1.81 \mathrm{e}-8$ & $2.00(1.50)$ \\
\cline { 2 - 9 } & $H^{\alpha / 2}$-norm & $6.24 \mathrm{e}-4$ & $2.43 \mathrm{e}-4$ & $9.54 \mathrm{e}-5$ & $3.77 \mathrm{e}-5$ & $1.49 \mathrm{e}-5$ & $5.91 \mathrm{e}-6$ & $2.35 \mathrm{e}-6$ & $1.34(1.33)$ \\
\hline
\end{tabular}

TABLE 3. The coefficient $\left({ }_{x} I_{1}^{\alpha} e\right)(0)$ for Riemann-Liouville case (respectively $\frac{1}{\Gamma(\alpha)}(x, e)$ for the Caputo case) in the adjoint solution representation for example (a), with $\alpha=3 / 2$, mesh size $h=1 /\left(2^{k} \times 10\right)$.

\begin{tabular}{|c|c|c|c|c|c|c|c|}
\hline$k$ & 1 & 2 & 3 & 4 & 5 & 6 & 7 \\
\hline R.-L. & $3.65 \mathrm{e}-3$ & $5.09 \mathrm{e}-4$ & $6.98 \mathrm{e}-5$ & $9.46 \mathrm{e}-6$ & $1.27 \mathrm{e}-6$ & $1.70 \mathrm{e}-7$ & $2.26 \mathrm{e}-8$ \\
Caputo & $1.76 \mathrm{e}-3$ & $2.24 \mathrm{e}-4$ & $2.85 \mathrm{e}-5$ & $3.59 \mathrm{e}-6$ & $4.53 \mathrm{e}-7$ & $5.69 \mathrm{e}-8$ & $7.13 \mathrm{e}-9$ \\
\hline
\end{tabular}

7.1.2. Numerical results for example (b). In this example, the source term $f$ is smooth, but does not satisfy the zero boundary condition. It belongs to $\widetilde{H}^{s}(D)$, $s \in[0,1 / 2)$. The exact solution $u$ is given by

$$
u(x)=\left\{\begin{aligned}
c_{\alpha}\left(x^{\alpha-1}-x^{\alpha}\right), & \text { Riemann-Liouville case } \\
c_{\alpha}\left(x-x^{\alpha}\right), & \text { Caputo case }
\end{aligned}\right.
$$

with the constant $c_{\alpha}=\frac{1}{\Gamma(\alpha+1)}$. The numerical results are shown in Tables 4 and 5 for the Riemann-Liouville and Caputo case, respectively. In the RiemannLiouville case, the convergence rates are identical with that for example (a). This is attributed to the fact that the regularity of the solution $u$ generally cannot go beyond $\widetilde{H}_{L}^{\alpha-1+\beta}(D)$ for $\beta \in(1-\alpha / 2,1 / 2)$. In contrast, in the Caputo case, for $\alpha \geq$ $3 / 2$, we observe identical convergence rates as example (a), whereas for $\alpha=4 / 3$, the convergence is slower due to limited smoothing induced by the fractional differential operator. As before, for either fractional derivative, the empirical convergence in $L^{2}(D)$-norm is better than the theoretical one by one-half order.

TABLE 4. Numerical results for example (b) with a RiemannLiouville fractional derivative, $f=1$, mesh size $h=1 /\left(2^{k} \times 10\right)$.

\begin{tabular}{|c|c|c|c|c|c|c|c|c|c|}
\hline$\alpha$ & $k$ & 1 & 2 & 3 & 4 & 5 & 6 & 7 & rate \\
\hline \multirow{3}{*}{$7 / 4$} & $L^{2}$-norm & $1.07 \mathrm{e}-3$ & $4.31 \mathrm{e}-4$ & $1.77 \mathrm{e}-4$ & $7.37 \mathrm{e}-5$ & $3.08 \mathrm{e}-5$ & $1.29 \mathrm{e}-5$ & $5.43 \mathrm{e}-6$ & $1.27(0.75)$ \\
\cline { 2 - 10 } & $H^{\alpha / 2}$-norm & $5.26 \mathrm{e}-2$ & $3.90 \mathrm{e}-2$ & $2.94 \mathrm{e}-2$ & $2.24 \mathrm{e}-2$ & $1.72 \mathrm{e}-2$ & $1.32 \mathrm{e}-2$ & $1.01 \mathrm{e}-2$ & $0.40(0.38)$ \\
\hline \multirow{3}{*}{$3 / 2$} & $L^{2}$-norm & $6.44 \mathrm{e}-3$ & $3.18 \mathrm{e}-3$ & $1.58 \mathrm{e}-3$ & $7.89 \mathrm{e}-4$ & $3.94 \mathrm{e}-4$ & $1.97 \mathrm{e}-4$ & $9.84 \mathrm{e}-5$ & $1.01(0.50)$ \\
\cline { 2 - 10 } & $H^{\alpha / 2}$-norm & $1.69 \mathrm{e}-1$ & $1.40 \mathrm{e}-1$ & $1.17 \mathrm{e}-1$ & $9.82 \mathrm{e}-2$ & $8.22 \mathrm{e}-2$ & $6.87 \mathrm{e}-2$ & $5.73 \mathrm{e}-2$ & $0.26(0.25)$ \\
\hline \multirow{2}{*}{$4 / 3$} & $L^{2}$-norm & $2.05 \mathrm{e}-2$ & $1.15 \mathrm{e}-2$ & $6.42 \mathrm{e}-3$ & $3.60 \mathrm{e}-3$ & $2.02 \mathrm{e}-3$ & $1.13 \mathrm{e}-3$ & $6.35 \mathrm{e}-4$ & $0.84(0.33)$ \\
\cline { 2 - 9 } & $H^{\alpha / 2}$-norm & $3.17 \mathrm{e}-1$ & $2.80 \mathrm{e}-1$ & $2.48 \mathrm{e}-1$ & $2.20 \mathrm{e}-1$ & $1.94 \mathrm{e}-1$ & $1.71 \mathrm{e}-1$ & $1.50 \mathrm{e}-1$ & $0.18(0.17)$ \\
\hline
\end{tabular}


TABLE 5. Numerical results for example (b) with a Caputo fractional derivative, $f=1$, mesh size $h=1 /\left(2^{k} \times 10\right)$.

\begin{tabular}{|c|c|c|c|c|c|c|c|c|c|}
\hline$\alpha$ & $k$ & 1 & 2 & 3 & 4 & 5 & 6 & 7 & rate \\
\hline \multirow{3}{*}{$7 / 4$} & $L^{2}$-norm & $1.74 \mathrm{e}-4$ & $4.21 \mathrm{e}-5$ & $1.03 \mathrm{e}-5$ & $2.51 \mathrm{e}-6$ & $6.16 \mathrm{e}-7$ & $1.51 \mathrm{e}-7$ & $3.74 \mathrm{e}-8$ & $2.00(1.50)$ \\
\cline { 2 - 10 } & $H^{\alpha / 2}$-norm & $8.14 \mathrm{e}-3$ & $3.74 \mathrm{e}-3$ & $1.72 \mathrm{e}-3$ & $7.91 \mathrm{e}-4$ & $3.63 \mathrm{e}-4$ & $1.67 \mathrm{e}-4$ & $7.65 \mathrm{e}-5$ & $1.12(1.13)$ \\
\hline \multirow{3}{*}{$3 / 2$} & $L^{2}$-norm & $1.88 \mathrm{e}-4$ & $4.84 \mathrm{e}-5$ & $1.24 \mathrm{e}-5$ & $3.17 \mathrm{e}-6$ & $8.12 \mathrm{e}-7$ & $2.07 \mathrm{e}-7$ & $5.29 \mathrm{e}-8$ & $1.97(1.50)$ \\
\cline { 2 - 10 } & $H^{\alpha / 2}$-norm & $4.81 \mathrm{e}-3$ & $2.12 \mathrm{e}-3$ & $9.33 \mathrm{e}-4$ & $4.08 \mathrm{e}-4$ & $1.78 \mathrm{e}-4$ & $7.76 \mathrm{e}-5$ & $3.37 \mathrm{e}-5$ & $1.20(1.25)$ \\
\hline \multirow{2}{*}{$4 / 3$} & $L^{2}$-norm & $2.48 \mathrm{e}-4$ & $6.99 \mathrm{e}-5$ & $1.97 \mathrm{e}-5$ & $5.53 \mathrm{e}-6$ & $1.55 \mathrm{e}-6$ & $4.36 \mathrm{e}-7$ & $1.22 \mathrm{e}-7$ & $1.83(1.33)$ \\
\cline { 2 - 9 } & $H^{\alpha / 2}$-norm & $3.44 \mathrm{e}-3$ & $1.55 \mathrm{e}-3$ & $6.96 \mathrm{e}-4$ & $3.12 \mathrm{e}-4$ & $1.40 \mathrm{e}-4$ & $6.26 \mathrm{e}-5$ & $2.80 \mathrm{e}-5$ & $1.16(1.17)$ \\
\hline
\end{tabular}

7.1.3. Numerical results for example (c). In this example, the source term $f$ is singular at the origin, and it belongs to the space $\widetilde{H}^{s}(D)$ for any $s \in[0,1 / 4)$. The exact solution $u$ is given by

$$
u(x)=\left\{\begin{aligned}
c_{\alpha}\left(x^{\alpha-1}-x^{\alpha-1 / 4}\right), & \text { Riemann-Liouville case, } \\
c_{\alpha}\left(x-x^{\alpha-1 / 4}\right), & \text { Caputo case }
\end{aligned}\right.
$$

with the constant $c_{\alpha}=\frac{\Gamma(3 / 4)}{\Gamma(\alpha+3 / 4)}$. The numerical results are shown in Tables 6 and 7 In the Riemann-Liouville case, the same convergence rates are observed (cf. Tables 1 and 4), concurring with Remark 4.2 and earlier observations. In the Caputo case, due to the lower regularity of the source term $f$, the numerical solution $u_{h}$ converges slower as the fractional order $\alpha$ approaches 1 , but the empirical convergence behavior still agrees well with the theoretical prediction.

TABLE 6. Numerical results for example (c) with a Riemann-

Liouville fractional derivative, $f=x^{-1 / 4}$, mesh size $h=1 /\left(2^{k} \times 10\right)$.

\begin{tabular}{|c|c|c|c|c|c|c|c|c|c|}
\hline$\alpha$ & $k$ & 1 & 2 & 3 & 4 & 5 & 6 & 7 & rate \\
\hline \multirow{3}{*}{$7 / 4$} & $L^{2}$-norm & $1.65 \mathrm{e}-3$ & $6.61 \mathrm{e}-4$ & $2.69 \mathrm{e}-4$ & $1.11 \mathrm{e}-4$ & $4.62 \mathrm{e}-5$ & $1.93 \mathrm{e}-5$ & $8.09 \mathrm{e}-6$ & $1.28(0.75)$ \\
\cline { 2 - 10 } & $H^{\alpha / 2}$-norm & $8.07 \mathrm{e}-2$ & $5.94 \mathrm{e}-2$ & $4.45 \mathrm{e}-2$ & $3.38 \mathrm{e}-2$ & $2.57 \mathrm{e}-2$ & $1.97 \mathrm{e}-2$ & $1.51 \mathrm{e}-2$ & $0.40(0.38)$ \\
\hline \multirow{3}{*}{$3 / 2$} & $L^{2}$-norm & $9.31 \mathrm{e}-3$ & $4.60 \mathrm{e}-3$ & $2.29 \mathrm{e}-3$ & $1.14 \mathrm{e}-3$ & $5.68 \mathrm{e}-4$ & $2.83 \mathrm{e}-4$ & $1.41 \mathrm{e}-4$ & $1.01(0.50)$ \\
\cline { 2 - 10 } & $H^{\alpha / 2}$-norm & $2.44 \mathrm{e}-1$ & $2.03 \mathrm{e}-1$ & $1.69 \mathrm{e}-1$ & $1.41 \mathrm{e}-1$ & $1.18 \mathrm{e}-1$ & $9.89 \mathrm{e}-2$ & $8.25 \mathrm{e}-2$ & $0.26(0.25)$ \\
\hline \multirow{2}{*}{$4 / 3$} & $L^{2}$-norm & $2.88 \mathrm{e}-2$ & $1.61 \mathrm{e}-2$ & $9.02 \mathrm{e}-3$ & $5.06 \mathrm{e}-3$ & $2.84 \mathrm{e}-3$ & $1.59 \mathrm{e}-3$ & $8.93 \mathrm{e}-4$ & $0.84(0.33)$ \\
\cline { 2 - 9 } & $H^{\alpha / 2}$-norm & $4.44 \mathrm{e}-1$ & $3.94 \mathrm{e}-1$ & $3.49 \mathrm{e}-1$ & $3.09 \mathrm{e}-1$ & $2.73 \mathrm{e}-1$ & $2.41 \mathrm{e}-1$ & $2.11 \mathrm{e}-1$ & $0.18(0.17)$ \\
\hline
\end{tabular}

TABle 7. Numerical results for example (c) with a Caputo fractional derivative, $f=x^{-1 / 4}$, mesh size $h=1 /\left(2^{k} \times 10\right)$.

\begin{tabular}{|c|c|c|c|c|c|c|c|c|c|}
\hline$\alpha$ & $k$ & 1 & 2 & 3 & 4 & 5 & 6 & 7 & rate \\
\hline \multirow{3}{*}{$7 / 4$} & $L^{2}$-norm & $3.04 \mathrm{e}-4$ & $7.67 \mathrm{e}-5$ & $1.93 \mathrm{e}-5$ & $4.88 \mathrm{e}-6$ & $1.23 \mathrm{e}-6$ & $3.11 \mathrm{e}-7$ & $7.86 \mathrm{e}-8$ & $1.99(1.50)$ \\
\cline { 2 - 10 } & $H^{\alpha / 2}$-norm & $1.21 \mathrm{e}-2$ & $5.85 \mathrm{e}-3$ & $2.82 \mathrm{e}-3$ & $1.35 \mathrm{e}-3$ & $6.46 \mathrm{e}-4$ & $3.08 \mathrm{e}-4$ & $1.46 \mathrm{e}-4$ & $1.07(1.13)$ \\
\hline \multirow{3}{*}{$3 / 2$} & $L^{2}$-norm & $3.93 \mathrm{e}-4$ & $1.09 \mathrm{e}-4$ & $3.06 \mathrm{e}-5$ & $8.69 \mathrm{e}-6$ & $2.49 \mathrm{e}-6$ & $7.21 \mathrm{e}-7$ & $2.10 \mathrm{e}-7$ & $1.81(1.25)$ \\
\cline { 2 - 10 } & $H^{\alpha / 2}$-norm & $6.84 \mathrm{e}-3$ & $3.48 \mathrm{e}-3$ & $1.75 \mathrm{e}-3$ & $8.82 \mathrm{e}-4$ & $4.43 \mathrm{e}-4$ & $2.22 \mathrm{e}-4$ & $1.11 \mathrm{e}-4$ & $0.99(1.00)$ \\
\hline \multirow{2}{*}{$4 / 3$} & $L^{2}$-norm & $4.31 \mathrm{e}-4$ & $1.18 \mathrm{e}-4$ & $3.33 \mathrm{e}-5$ & $9.83 \mathrm{e}-6$ & $3.01 \mathrm{e}-6$ & $9.47 \mathrm{e}-7$ & $3.05 \mathrm{e}-7$ & $1.70(1.08)$ \\
\cline { 2 - 9 } & $H^{\alpha / 2}$-norm & $2.60 \mathrm{e}-3$ & $1.43 \mathrm{e}-3$ & $7.72 \mathrm{e}-4$ & $4.13 \mathrm{e}-4$ & $2.20 \mathrm{e}-4$ & $1.17 \mathrm{e}-4$ & $6.19 \mathrm{e}-5$ & $0.90(0.92)$ \\
\hline
\end{tabular}


7.2. Eigenvalue problem. In this part we illustrate the FSLP with the following three potentials:

(a) a zero potential $q_{1}=0$;

(b) a smooth potential $q_{2}=20 x^{3}(1-x) e^{-x}$;

(c) a discontinuous potential $q_{3}=-2 x \chi_{[0,1 / 5]}+(-4 / 5+2 x) \chi_{[1 / 5,2 / 5]}+\chi_{[3 / 5,4 / 5]}$.

The potentials $q_{1}$ and $q_{2}$ are smooth and belong to the space $\widetilde{H}^{1}(D)$, while the potential $q_{3}$ is piecewise smooth and belongs to the space $\widetilde{H}^{s}(D)$ for any $s<1 / 2$.

First, we note that the FSLP (1.2) is closely connected with the Mittag-Leffler function 21]

$$
E_{\alpha, \beta}(z)=\sum_{k=0}^{\infty} \frac{z^{k}}{\Gamma(k \alpha+\beta)}, \quad z \in \mathbb{C} .
$$

It is well known that problem (1.2) for $q(x) \equiv 0$ has infinitely many eigenvalues $\lambda$ that are zeros of the Mittag-Leffler functions $E_{\alpha, 2}(-\lambda)$ and $E_{\alpha, \alpha}(-\lambda)$ for the Caputo and Riemann-Liouville derivatives, respectively (see [12,24 for related discussions). This can be numerically verified directly; cf. Figure1. However, computing zeros of the Mittag-Leffler function in a stable and accurate way remains a very challenging task (in fact, evaluating the Mittag-Leffler function $E_{\alpha, \beta}(z)$ to a high accuracy is already highly nontrivial [16, 17, 30]), and further, it does not cover the interesting case of a general potential $q$.
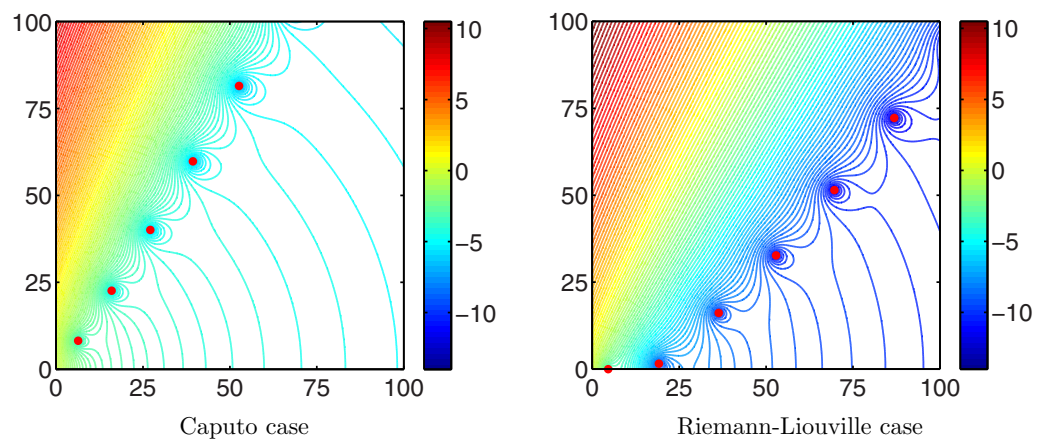

FiguRE 1. Zeros of Mittag-Leffler function $E_{\alpha, \beta}(-\lambda)$ and the variational eigenvalues for $q=0$ for $\alpha=4 / 3$. The red dots are the eigenvalues computed by the finite element method, and the contour lines plot the value of the functions $\log \left|E_{\alpha, 2}(-\lambda)\right|$ (left) and $\log \left|E_{\alpha, \alpha}(-\lambda)\right|$ (right) over the complex domain. The red dots lie in the deep blue wells, which correspond to zeros of the Mittag-Leffler functions.

As before, we divide the domain $D=(0,1)$ into a uniform mesh with a mesh size $h$. We measure the accuracy of an FEM approximation $\lambda_{h}$, by the absolute error, i.e., $e\left(\lambda_{h}\right)=\left|\lambda-\lambda_{h}\right|$ and the reference value $\lambda$ is computed on a very refined mesh with $h=1 / 10240$ and checked against that computed by the quasi-Newton method developed in [20]. The resulting discrete eigenvalue problems were solved by built-in MATLAB function eigs with a default tolerance. Below we shall discuss the Caputo and Riemann-Liouville cases separately, since their eigenfunctions have 
very different regularity, and one naturally expects that the approximations exhibit different convergence behavior.

7.2.1. Caputo derivative case. By the solution theory in Section 4, for the potentials $q_{1}$ and $q_{2}$, the eigenfunctions are in the space $H^{\alpha+1}(D) \cap \widetilde{H}^{\alpha / 2}(D)$, whereas for the potential $q_{3}$, the eigenfunctions lie in the space $H^{\alpha+s}(D) \cap \widetilde{H}^{\alpha / 2}(D)$ for any $s \in[0,1 / 2)$. Hence they can be well approximated on uniform meshes. Further, the regularity predicts a convergence rate $O\left(h^{\min (\alpha+s, 2)-1 / 2}\right)(s \in[0,1]$ is an exponent such that $\left.q \in \widetilde{H}^{s}(D)\right)$ for the approximate eigenvalues, with a best possible rate $O\left(h^{3 / 2}\right)$.

In Tables 8 10 we present the errors of the first ten eigenvalues for $\alpha=5 / 3$, where the empirical convergence rates are also shown. For all three potentials, there are only two real eigenvalues, and the rest appears as complex conjugate pairs. All the computed eigenvalues are simple. Numerically, a second-order convergence is observed for all the eigenvalues. The presence of a potential term influences the errors very little: the errors are almost identical for all three potentials. The empirical rate is one-half order higher than the theoretical one. The mechanism of "superconvergence" still awaits explanation.

These observations were also confirmed by the numerical results for other $\alpha$ values; see Figure 2 for an illustration for the potential $q_{3}$. As the $\alpha$ value increases towards two, the number of real eigenvalues (which appear always in pair) also increases accordingly. The overall convergence seems relatively independent of the $\alpha$ values, except for $\alpha=7 / 4$, for which there are four real eigenvalues. Here the convergence rates for the first and second eigenvalues are different, with one slightly below two and the other slightly above two; see also Table 11 A similar but less dramatic difference can be observed for the third and fourth eigenvalues. The rest of the eigenvalues exhibits a second-order convergence. Hence the abnormality is only observed for the "newly" emerged real eigenvalues.
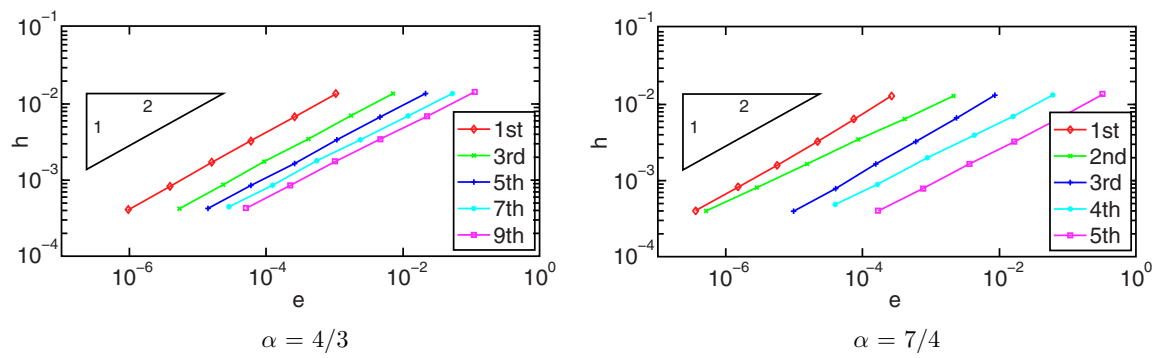

FIgURE 2. The convergence of the finite element approximations of the eigenvalues with the potential $q_{3}$, for Caputo derivative with $\alpha=4 / 3$, and $7 / 4$.

The Caputo eigenfunctions are fairly regular. We illustrate this in Figure 3 , where the first, fifth and tenth eigenfunctions for two different $\alpha$ values, i.e., $4 / 3$, and $5 / 3$, were shown. The eigenfunctions are normalized to have a unit $L^{2}(D)$-norm. The profiles of the eigenfunctions generally resemble sinusoidal functions, but the magnitudes are attenuated around $x=1$, which is related to the asymptotics of the Mittag-Leffler function $x E_{\alpha, 2}\left(-\lambda_{n} x^{\alpha}\right)$, the eigenfunction in the case of a zero potential. The degree of attenuation depends on the order $\alpha$. Further, the larger 
TABLE 8. The errors of the first ten eigenvalues for $\alpha=5 / 3, q_{1}$, Caputo derivative, mesh size $h=1 /\left(10 \times 2^{k}\right), k=3, \ldots, 8$.

\begin{tabular}{cccccccc}
\hline$e \backslash k$ & 3 & 4 & 5 & 6 & 7 & 8 & rate \\
\hline$\lambda_{1}$ & $1.03 \mathrm{e}-3$ & $2.55 \mathrm{e}-4$ & $6.33 \mathrm{e}-5$ & $1.57 \mathrm{e}-5$ & $3.89 \mathrm{e}-6$ & $9.37 \mathrm{e}-7$ & 2.01 \\
$\lambda_{2}$ & $1.64 \mathrm{e}-3$ & $3.33 \mathrm{e}-4$ & $6.80 \mathrm{e}-5$ & $1.39 \mathrm{e}-5$ & $2.87 \mathrm{e}-6$ & $6.02 \mathrm{e}-7$ & 2.28 \\
$\lambda_{3,4}$ & $3.75 \mathrm{e}-2$ & $8.19 \mathrm{e}-3$ & $1.82 \mathrm{e}-3$ & $4.11 \mathrm{e}-4$ & $9.35 \mathrm{e}-5$ & $2.07 \mathrm{e}-5$ & 2.15 \\
$\lambda_{5,6}$ & $1.58 \mathrm{e}-1$ & $3.32 \mathrm{e}-2$ & $7.08 \mathrm{e}-3$ & $1.53 \mathrm{e}-3$ & $3.34 \mathrm{e}-4$ & $7.18 \mathrm{e}-5$ & 2.20 \\
$\lambda_{7,8}$ & $4.87 \mathrm{e}-1$ & $1.00 \mathrm{e}-1$ & $2.09 \mathrm{e}-2$ & $4.40 \mathrm{e}-3$ & $9.35 \mathrm{e}-4$ & $1.95 \mathrm{e}-4$ & 2.24 \\
$\lambda_{9,10}$ & $1.18 \mathrm{e} 0$ & $2.39 \mathrm{e}-1$ & $4.92 \mathrm{e}-2$ & $1.02 \mathrm{e}-2$ & $2.13 \mathrm{e}-3$ & $4.37 \mathrm{e}-4$ & 2.27 \\
\hline
\end{tabular}

TABLE 9. The errors of the first ten eigenvalues for $\alpha=5 / 3, q_{2}$, Caputo derivative, mesh size $h=1 /\left(10 \times 2^{k}\right), k=3, \ldots, 8$.

\begin{tabular}{cccccccc}
\hline$e \backslash k$ & 3 & 4 & 5 & 6 & 7 & 8 & rate \\
\hline$\lambda_{1}$ & $1.11 \mathrm{e}-3$ & $2.75 \mathrm{e}-4$ & $6.79 \mathrm{e}-5$ & $1.68 \mathrm{e}-5$ & $4.14 \mathrm{e}-6$ & $9.95 \mathrm{e}-7$ & 2.02 \\
$\lambda_{2}$ & $1.75 \mathrm{e}-3$ & $3.59 \mathrm{e}-4$ & $7.38 \mathrm{e}-5$ & $1.53 \mathrm{e}-5$ & $3.18 \mathrm{e}-6$ & $6.74 \mathrm{e}-7$ & 2.27 \\
$\lambda_{3,4}$ & $3.75 \mathrm{e}-2$ & $8.21 \mathrm{e}-3$ & $1.83 \mathrm{e}-3$ & $4.13 \mathrm{e}-4$ & $9.39 \mathrm{e}-5$ & $2.08 \mathrm{e}-5$ & 2.15 \\
$\lambda_{5,6}$ & $1.58 \mathrm{e}-1$ & $3.33 \mathrm{e}-2$ & $7.09 \mathrm{e}-3$ & $1.53 \mathrm{e}-3$ & $3.35 \mathrm{e}-4$ & $7.20 \mathrm{e}-5$ & 2.21 \\
$\lambda_{7,8}$ & $4.87 \mathrm{e}-1$ & $1.00 \mathrm{e}-1$ & $2.09 \mathrm{e}-2$ & $4.40 \mathrm{e}-3$ & $9.35 \mathrm{e}-4$ & $1.96 \mathrm{e}-4$ & 2.24 \\
$\lambda_{9,10}$ & $1.18 \mathrm{e} 0$ & $2.39 \mathrm{e}-1$ & $4.92 \mathrm{e}-2$ & $1.02 \mathrm{e}-2$ & $2.13 \mathrm{e}-3$ & $4.37 \mathrm{e}-4$ & 2.27 \\
\hline
\end{tabular}

TABLE 10. The error of the first ten eigenvalues for $\alpha=5 / 3, q_{3}$, Caputo derivative, mesh size $h=1 /\left(10 \times 2^{k}\right), k=3, \ldots, 8$.

\begin{tabular}{cccccccc}
\hline$e \backslash k$ & 3 & 4 & 5 & 6 & 7 & 8 & rate \\
\hline$\lambda_{1}$ & $1.10 \mathrm{e}-3$ & $2.71 \mathrm{e}-4$ & $6.69 \mathrm{e}-5$ & $1.66 \mathrm{e}-5$ & $4.08 \mathrm{e}-6$ & $9.81 \mathrm{e}-7$ & 2.02 \\
$\lambda_{2}$ & $1.73 \mathrm{e}-3$ & $3.53 \mathrm{e}-4$ & $7.23 \mathrm{e}-5$ & $1.48 \mathrm{e}-5$ & $3.08 \mathrm{e}-6$ & $6.47 \mathrm{e}-7$ & 2.27 \\
$\lambda_{3,4}$ & $3.79 \mathrm{e}-2$ & $8.31 \mathrm{e}-3$ & $1.85 \mathrm{e}-3$ & $4.18 \mathrm{e}-4$ & $9.52 \mathrm{e}-5$ & $2.11 \mathrm{e}-5$ & 2.14 \\
$\lambda_{5,6}$ & $1.58 \mathrm{e}-1$ & $3.33 \mathrm{e}-2$ & $7.09 \mathrm{e}-3$ & $1.53 \mathrm{e}-3$ & $3.35 \mathrm{e}-4$ & $7.20 \mathrm{e}-5$ & 2.20 \\
$\lambda_{7,8}$ & $4.87 \mathrm{e}-1$ & $1.00 \mathrm{e}-1$ & $2.08 \mathrm{e}-2$ & $4.40 \mathrm{e}-3$ & $9.34 \mathrm{e}-4$ & $1.95 \mathrm{e}-4$ & 2.24 \\
$\lambda_{9,10}$ & $1.18 \mathrm{e} 0$ & $2.39 \mathrm{e}-1$ & $4.92 \mathrm{e}-2$ & $1.02 \mathrm{e}-2$ & $2.13 \mathrm{e}-3$ & $4.37 \mathrm{e}-4$ & 2.27 \\
\hline
\end{tabular}

TABLE 11. The errors of the first ten eigenvalues for $\alpha=7 / 4, q_{3}$, Caputo derivative, mesh size $h=1 /\left(10 \times 2^{k}\right), k=3, \ldots, 8$.

\begin{tabular}{cccccccc}
\hline$e \backslash k$ & 3 & 4 & 5 & 6 & 7 & 8 & rate \\
\hline$\lambda_{1}$ & $2.79 \mathrm{e}-4$ & $7.84 \mathrm{e}-5$ & $2.14 \mathrm{e}-5$ & $5.73 \mathrm{e}-6$ & $1.50 \mathrm{e}-6$ & $3.74 \mathrm{e}-7$ & 1.92 \\
$\lambda_{2}$ & $2.19 \mathrm{e}-3$ & $4.30 \mathrm{e}-4$ & $8.27 \mathrm{e}-5$ & $1.54 \mathrm{e}-5$ & $2.77 \mathrm{e}-6$ & $4.79 \mathrm{e}-7$ & 2.40 \\
$\lambda_{3}$ & $8.63 \mathrm{e}-3$ & $2.29 \mathrm{e}-3$ & $6.05 \mathrm{e}-4$ & $1.58 \mathrm{e}-4$ & $4.06 \mathrm{e}-5$ & $9.85 \mathrm{e}-6$ & 1.95 \\
$\lambda_{4}$ & $5.89 \mathrm{e}-2$ & $1.26 \mathrm{e}-2$ & $2.71 \mathrm{e}-3$ & $5.80 \mathrm{e}-4$ & $1.24 \mathrm{e}-4$ & $2.57 \mathrm{e}-5$ & 2.22 \\
$\lambda_{5,6}$ & $3.44 \mathrm{e}-1$ & $7.44 \mathrm{e}-2$ & $1.63 \mathrm{e}-2$ & $3.60 \mathrm{e}-3$ & $7.96 \mathrm{e}-4$ & $1.72 \mathrm{e}-4$ & 2.18 \\
$\lambda_{7,8}$ & $8.46 \mathrm{e}-1$ & $1.81 \mathrm{e}-1$ & $3.89 \mathrm{e}-2$ & $8.41 \mathrm{e}-3$ & $1.81 \mathrm{e}-3$ & $3.82 \mathrm{e}-4$ & 2.21 \\
$\lambda_{9,10}$ & $2.06 \mathrm{e} 0$ & $4.38 \mathrm{e}-1$ & $9.35 \mathrm{e}-2$ & $2.00 \mathrm{e}-2$ & $4.28 \mathrm{e}-3$ & $8.93 \mathrm{e}-4$ & 2.22 \\
\hline
\end{tabular}

is the eigenvalue, the more oscillatory is the corresponding eigenfunction, and the number of interior zero differs by one for the real and imaginary parts of a genuinely complex eigenfunction. 

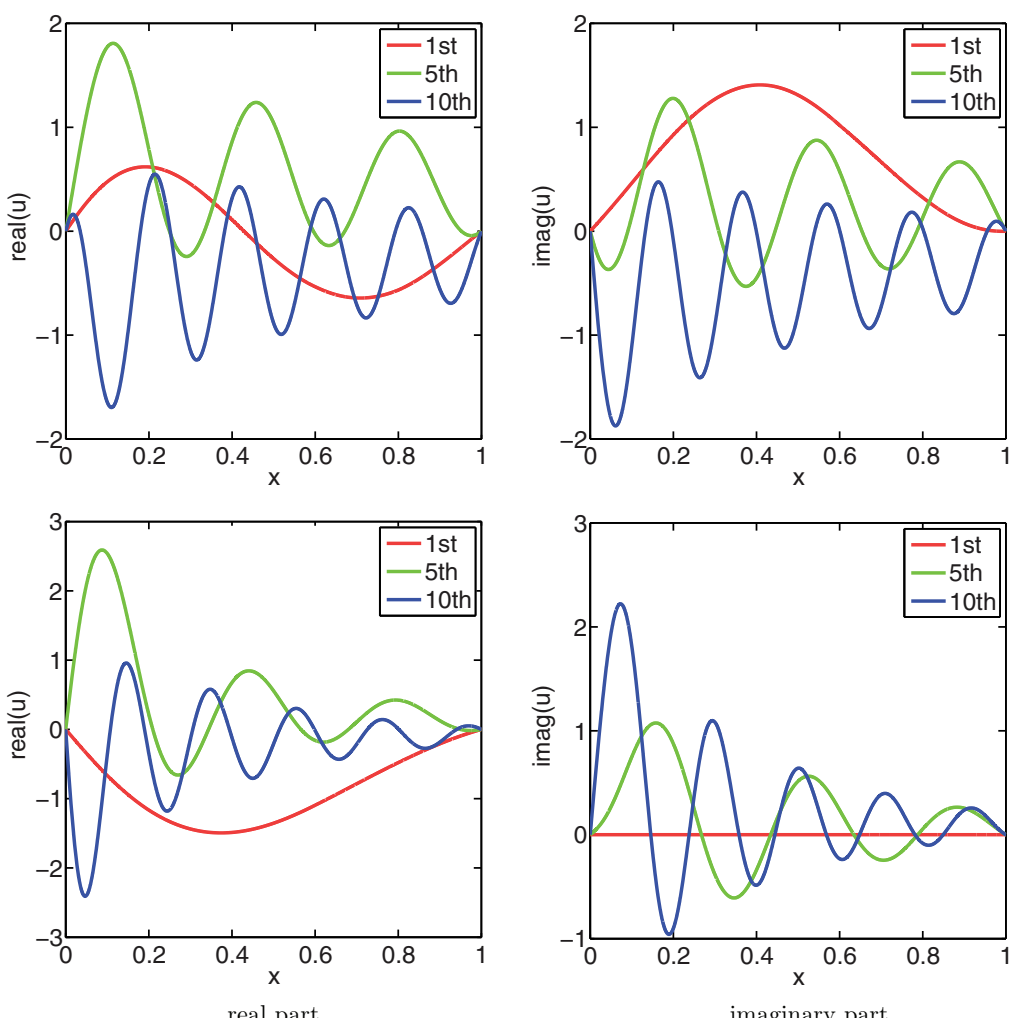

Figure 3. The profile of the first, fifth and tenth eigenfunctions in case of $q_{3}, \alpha=4 / 3$ (top) and $\alpha=5 / 3$ (bottom), Caputo case.

7.2.2. Riemann-Liouville derivative case. By the regularity theory in Section 4 in the Riemann-Liouville case, the eigenfunctions are less regular, with an inherent singularity of the form $x^{\alpha-1}$ concentrated at the origin. Hence, one would naturally expect a slow convergence of the finite element approximations with a uniform mesh. Our experiments indicate that finite element approximations of the eigenfunctions for $\alpha$ close to unity indeed suffer from oscillations near the origin, and thus converge much slower. The finite element method does converge, and hence the oscillations go away as the mesh refines.

The numerical results for $\alpha=5 / 3$ with the three potentials are presented in Tables 12 14. For all three potentials, the first nine eigenvalues are real (see Appendix A for the existence of one real eigenvalue) and the rest appears as conjugate pairs, and in the tables, we show the convergence results only for the first six eigenvalues. Surprisingly, the eigenvalue approximations still exhibit a second-order convergence. As before, the convergence rate is almost independent of the potential term. The preceding observation remains largely true, except within a "transient" region $1.70 \leq \alpha \leq 1.85$ to which the value $\alpha=7 / 4$ belongs: the method still converges almost at the same rate, but the convergence is not as steady as for other cases. However, although not presented, we would like to remark that the convergence for larger eigenvalues is rather steady. 
TABLE 12. The errors of the first six eigenvalues, which are all real, for $\alpha=5 / 3, q_{1}$, Riemann-Liouville derivative, mesh size $h=$ $1 /\left(10 \times 2^{k}\right), k=3, \ldots, 8$.

\begin{tabular}{cccccccc}
\hline$e \backslash k$ & 3 & 4 & 5 & 6 & 7 & 8 & rate \\
\hline$\lambda_{1}$ & $3.53 \mathrm{e}-4$ & $9.36 \mathrm{e}-5$ & $2.44 \mathrm{e}-5$ & $6.31 \mathrm{e}-6$ & $1.60 \mathrm{e}-6$ & $3.95 \mathrm{e}-7$ & 1.95 \\
$\lambda_{2}$ & $2.73 \mathrm{e}-3$ & $7.30 \mathrm{e}-4$ & $1.92 \mathrm{e}-4$ & $4.98 \mathrm{e}-5$ & $1.27 \mathrm{e}-5$ & $3.09 \mathrm{e}-6$ & 1.96 \\
$\lambda_{3}$ & $7.33 \mathrm{e}-3$ & $1.99 \mathrm{e}-3$ & $5.32 \mathrm{e}-4$ & $1.39 \mathrm{e}-4$ & $3.58 \mathrm{e}-5$ & $8.64 \mathrm{e}-6$ & 1.96 \\
$\lambda_{4}$ & $1.81 \mathrm{e}-2$ & $4.80 \mathrm{e}-3$ & $1.27 \mathrm{e}-3$ & $3.31 \mathrm{e}-4$ & $8.46 \mathrm{e}-5$ & $2.05 \mathrm{e}-5$ & 1.96 \\
$\lambda_{5}$ & $2.62 \mathrm{e}-2$ & $7.11 \mathrm{e}-3$ & $1.93 \mathrm{e}-3$ & $5.16 \mathrm{e}-4$ & $1.35 \mathrm{e}-4$ & $3.39 \mathrm{e}-5$ & 1.95 \\
$\lambda_{6}$ & $6.59 \mathrm{e}-2$ & $1.65 \mathrm{e}-2$ & $4.24 \mathrm{e}-3$ & $1.09 \mathrm{e}-3$ & $2.77 \mathrm{e}-4$ & $6.81 \mathrm{e}-5$ & 1.98 \\
\hline
\end{tabular}

TABLE 13. The errors of the first six eigenvalues, which are all real, for $\alpha=5 / 3, q_{2}$, Riemann-Liouville derivative, mesh size $h=$ $1 /\left(10 \times 2^{k}\right), k=3, \ldots, 8$.

\begin{tabular}{cccccccc}
\hline$e \backslash k$ & 3 & 4 & 5 & 6 & 7 & 8 & rate \\
\hline$\lambda_{1}$ & $3.68 \mathrm{e}-4$ & $9.78 \mathrm{e}-5$ & $2.56 \mathrm{e}-5$ & $6.61 \mathrm{e}-6$ & $1.68 \mathrm{e}-6$ & $4.13 \mathrm{e}-7$ & 1.96 \\
$\lambda_{2}$ & $2.78 \mathrm{e}-3$ & $7.43 \mathrm{e}-4$ & $1.96 \mathrm{e}-4$ & $5.08 \mathrm{e}-5$ & $1.29 \mathrm{e}-5$ & $3.14 \mathrm{e}-6$ & 1.96 \\
$\lambda_{3}$ & $7.41 \mathrm{e}-3$ & $2.01 \mathrm{e}-3$ & $5.38 \mathrm{e}-4$ & $1.41 \mathrm{e}-4$ & $3.62 \mathrm{e}-5$ & $8.75 \mathrm{e}-6$ & 1.95 \\
$\lambda_{4}$ & $1.82 \mathrm{e}-2$ & $4.83 \mathrm{e}-3$ & $1.27 \mathrm{e}-3$ & $3.33 \mathrm{e}-4$ & $8.52 \mathrm{e}-5$ & $2.06 \mathrm{e}-5$ & 1.95 \\
$\lambda_{5}$ & $2.63 \mathrm{e}-2$ & $7.15 \mathrm{e}-3$ & $1.94 \mathrm{e}-3$ & $5.20 \mathrm{e}-4$ & $1.36 \mathrm{e}-4$ & $3.41 \mathrm{e}-5$ & 1.94 \\
$\lambda_{6}$ & $6.60 \mathrm{e}-2$ & $1.65 \mathrm{e}-2$ & $4.25 \mathrm{e}-3$ & $1.09 \mathrm{e}-3$ & $2.78 \mathrm{e}-4$ & $6.83 \mathrm{e}-5$ & 1.97 \\
\hline
\end{tabular}

TABLE 14. The errors of the first six eigenvalues, which are all real, for $\alpha=5 / 3, q_{3}$, Riemann-Liouville derivative, mesh size $h=$ $1 /\left(10 \times 2^{k}\right), k=3, \ldots, 8$.

\begin{tabular}{cccccccc}
\hline$e \backslash k$ & 3 & 4 & 5 & 6 & 7 & 8 & rate \\
\hline$\lambda_{1}$ & $3.60 \mathrm{e}-4$ & $9.57 \mathrm{e}-5$ & $2.50 \mathrm{e}-5$ & $6.46 \mathrm{e}-6$ & $1.64 \mathrm{e}-6$ & $4.04 \mathrm{e}-7$ & 1.96 \\
$\lambda_{2}$ & $2.78 \mathrm{e}-3$ & $7.44 \mathrm{e}-4$ & $1.96 \mathrm{e}-4$ & $5.08 \mathrm{e}-5$ & $1.29 \mathrm{e}-5$ & $3.14 \mathrm{e}-6$ & 1.95 \\
$\lambda_{3}$ & $7.35 \mathrm{e}-3$ & $1.99 \mathrm{e}-3$ & $5.34 \mathrm{e}-4$ & $1.40 \mathrm{e}-4$ & $3.59 \mathrm{e}-5$ & $8.68 \mathrm{e}-6$ & 1.95 \\
$\lambda_{4}$ & $1.80 \mathrm{e}-2$ & $4.79 \mathrm{e}-3$ & $1.27 \mathrm{e}-3$ & $3.31 \mathrm{e}-4$ & $8.45 \mathrm{e}-5$ & $2.05 \mathrm{e}-5$ & 1.95 \\
$\lambda_{5}$ & $2.63 \mathrm{e}-2$ & $7.13 \mathrm{e}-3$ & $1.94 \mathrm{e}-3$ & $5.18 \mathrm{e}-4$ & $1.35 \mathrm{e}-4$ & $3.40 \mathrm{e}-5$ & 1.94 \\
$\lambda_{6}$ & $6.59 \mathrm{e}-2$ & $1.65 \mathrm{e}-2$ & $4.24 \mathrm{e}-3$ & $1.09 \mathrm{e}-3$ & $2.77 \mathrm{e}-4$ & $6.81 \mathrm{e}-5$ & 1.97 \\
\hline
\end{tabular}

Our theory predicts that the eigenfunctions for the Caputo and Riemann-Liouville derivative behave very differently. To confirm this, we plot eigenfunctions for the latter in Figure 4 . One can observe from Figures 3 and 4 that apart from a stronger singularity at the origin, the Riemann-Liouville eigenfunctions are also far more significantly attenuated towards $x=1$. In case of $q=0$, this can be explained by the exponential asymtotics of the Mittag-Leffler function: the eigenfunctions in the Caputo and Riemann-Liouville cases are given by $x E_{\alpha, 2}\left(-\lambda_{n} x^{\alpha}\right)$ and $x^{\alpha-1} E_{\alpha, \alpha}\left(-\lambda_{n} x^{\alpha}\right)$, respectively; the former decays only linearly, whereas the latter decays quadratically [21, pp. 43]; This can also be deduced from Figure1 the wells, which correspond to zeros of the Mittag-Leffler functions, run much deeper for Riemann-Liouville case. We refer interested readers to [19] for further numerical studies on the FSLP with a Riemann-Liouville derivative. 

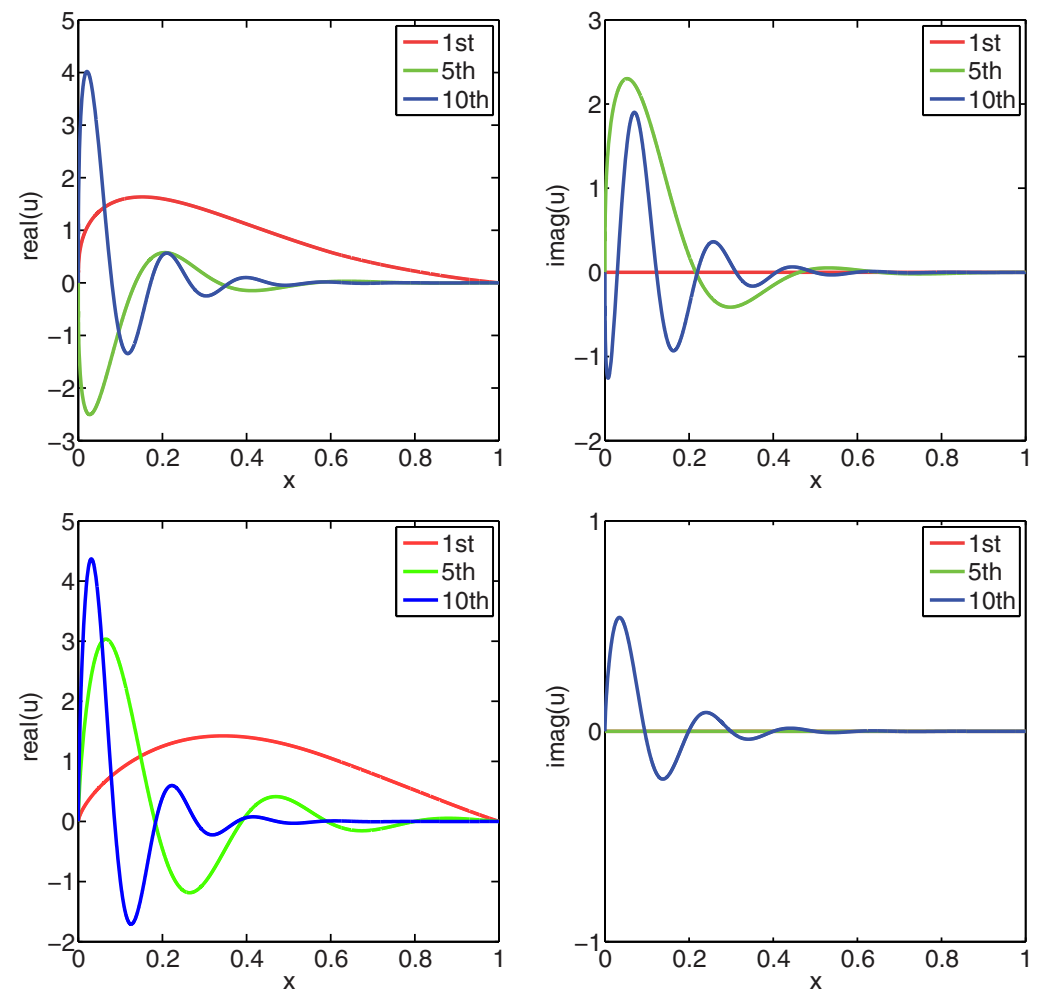

real part

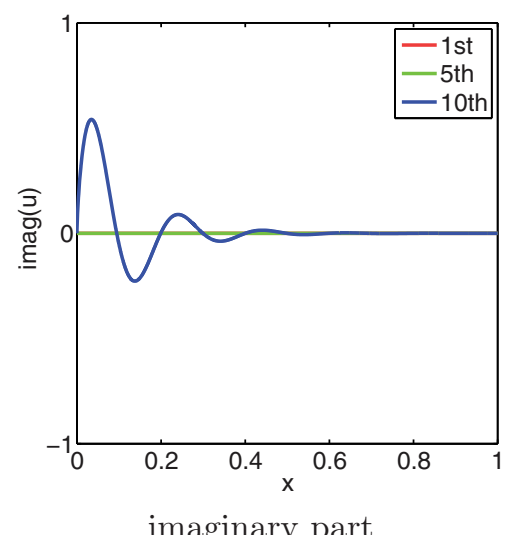

Figure 4. The profile of the first, fifth and tenth eigenfunctions in the case of $q_{3}, \alpha=4 / 3$ (top), $\alpha=5 / 3$ (bottom), Riemann-Liouville case.

7.2.3. Two extensions. In this part, we discuss two possible extensions of the finite element formulation.

A first natural idea of extension is to pursue other boundary conditions, e.g., Neumann, Robin or mixed type. The derivation of the variational formulation in Section 4 requires highly nontrivial modifications for these variations. As an illustration, we make the following straightforward attempt for the Riemann-Liouville case with mixed boundary conditions:

$$
-{ }_{0}^{R} D_{x}^{\alpha} u+q u=f \quad \text { in } D, \quad{ }_{0}^{R} D_{x}^{\alpha-1} u(0)=u(1)=0,
$$

where $f \in L^{2}(D)$. Let $g={ }_{0} I_{x}^{\alpha} f \in \widetilde{H}_{L}^{\alpha}(D)$, then ${ }_{0}^{R} D_{x}^{\alpha} g=f$. We thus find that

$$
u=-{ }_{0} I_{x}^{\alpha} f+\left({ }_{0} I_{x}^{\alpha} f\right)(1) x^{\alpha-2}
$$

is a solution of (7.2) in the Riemann-Louiville case (for $q=0$ ) since it satisfies the boundary conditions and ${ }_{0}^{R} D_{x}^{\alpha} x^{\alpha-2}=\left(c_{\alpha}\right)^{\prime \prime}=0$. The representation formula is very suggestive. First, the most "natural" variational formulation for (7.2), i.e., find $u \in \widetilde{H}_{R}^{\alpha / 2}(D)$ such that

$$
a(u, v)=(f, v) \quad \forall v \in \widetilde{H}_{R}^{\alpha / 2}(D),
$$


with a bilinear form $a(u, v)=-\left({ }_{0}^{R} D_{x}^{\alpha / 2} u,{ }_{x}^{R} D_{1}^{\alpha / 2} v\right)+(q u, v)$, cannot be stable on the space $\widetilde{H}_{R}^{\alpha / 2}(D)$, since in general the solution $u$ does not admit the $\widetilde{H}_{R}^{\alpha / 2}(D)$ regularity, due to the presence of the singular term $x^{\alpha-2}$. Hence, alternative formulations, e.g., of Petrov-Galerkin type, should be sought for. Further, the solution $u$ can have only very limited Sobolev regularity, and a special treatment at the left end point is necessary. This clearly illustrates the delicacy of properly treating boundary conditions.

We have focused our discussions on the left-sided Riemann-Liouville and Caputo derivatives. There are several alternative choices of the spatial derivative, depending on the specific applications. For example, one may also consider a mixed derivative $\mathbf{D}_{\theta}^{\alpha}$ defined by

$$
\mathbf{D}_{\theta}^{\alpha} u=\theta_{0}^{R} D_{x}^{\alpha} u+(1-\theta){ }_{x}^{R} D_{1}^{\alpha} u \quad \text { or } \quad \mathbf{D}_{\theta}^{\alpha} u=\theta_{0}^{C} D_{x}^{\alpha} u+(1-\theta){ }_{x}^{C} D_{1}^{\alpha} u,
$$

where $\theta \in[0,1]$ is a skewness parameter. The mixed derivative has been very popular in modeling superdiffusion process. For such mixed derivative, there is no known variational formulation, solution representation formula and regularity pickup. Formally, for the fractional SLP with a mixed Riemann-Liouville derivative and $u(0)=u(1)=0$, one would expect that the respective weak formulation reads: find $u \in \widetilde{H}^{\alpha / 2}(D)$ and $\lambda \in \mathbb{C}$ such that

$$
\theta\left({ }_{0}^{R} D_{x}^{\alpha / 2} u,{ }_{x}^{R} D_{1}^{\alpha / 2} v\right)+(1-\theta)\left({ }_{x}^{R} D_{1}^{\alpha / 2} u,{ }_{0}^{R} D_{x}^{\alpha / 2} v\right)=\lambda(u, v), \quad \text { for all } v \in \widetilde{H}^{\alpha / 2}(D) .
$$

However, it is still unclear whether this does represent the proper variational formulation, due to a lack of the solution regularity, especially around the end points. Our numerical experiments with this formulation indicate that the eigenfunctions have singularity only at one end point, depending on the value of the parameter $\theta$ : for $\theta>1 / 2$, the singularity is at the left end point, whereas for $\theta<1 / 2$, it is at the right end point. Due to the presence of fractional derivatives from both end points, the presence of only one single singularity seems counterintuitive. Nonetheless, in view of the empirically observed solution regularity, the numerical experiments do confirm a posteriori that the variational formulation in the Riemann-Liouville case seems plausible. However, a complete mathematical justification of the formulation is still missing. Further, the case of a mixed Caputo derivative is completely unclear.

\section{Concluding Remarks}

In this work we have developed variational formulations for boundary value problems involving either Riemann-Liouville or Caputo fractional derivatives of order $\alpha \in(1,2)$. The stability of the variational formulations, and the Sobolev regularity of the variational solutions were established. Moreover, the finite element discretization of the scheme was developed, and convergence rates in $\widetilde{H}^{\alpha / 2}(D)$ - and $L^{2}(D)$-norms were established. The finite element method has been applied to the fractional Sturm-Liouville problem, and (suboptimal) convergence rates were obtained. For the source problem, the error estimates in $\widetilde{H}^{\alpha / 2}(D)$-norm were fully supported by the numerical experiments, whereas the $L^{2}(D)$-estimates remain onehalf order lower than the empirical convergence rates, which requires further investigations. For the eigenvalue problem, it converges at a second-order rate for both fractional derivatives, and can provide accurate estimates of multiple eigenvalues in the presence of either a smooth or nonsmooth potential term. 
There are several avenues for future works. First, in the Riemann-Liouville case, the solution generally contains a singularity of the form $x^{\alpha-1}$, and numerically on a uniform mesh we have observed that the numerical solutions suffer from spurious oscillations around the origin, especially for $\alpha$ close to unity. This necessitates the use of an adaptively refined mesh or an enriched solution space. Second, it is natural to extend the analysis to the parabolic counterpart. Third, there are many possible extensions, e.g., mixed left-sided and right-sided Caputo/RiemannLiouville fractional derivatives and different boundary conditions. However, the proper variational formulation and solution theory, especially regularity pickup, for such models are still unclear. Last, the theoretical justification of the superior performance of the finite element method for the eigenvalue problem is of immense interest.

\section{Appendix A. Existence of a ReAl eigenvalue}

In this appendix, we show that the lowest Dirichlet eigenvalue in the case of a Riemann-Liouville derivative, with a zero potential, is always positive. To this end, we consider the solution operator $T: C_{0}(D) \rightarrow C_{0}(D), f \rightarrow T f$, with $T f$ defined by

$$
T f=\left({ }_{0} I_{x}^{\alpha} f\right)(1) x^{\alpha-1}-{ }_{0} I_{x}^{\alpha} f(x) .
$$

By Theorem 4.4, the operator $T: C_{0}(D) \rightarrow C_{0}(D)$ is compact. Let $K$ be the set of nonnegative functions in $C_{0}(D)$. Next we show that the operator $T$ is positive on $K$. Let $f \in C_{0}(D)$, and $f \geq 0$. Then

$$
\begin{aligned}
T f(x)= & \frac{1}{\Gamma(\alpha)} \int_{0}^{1}(1-t)^{\alpha-1} f(t) d t x^{\alpha-1}-\frac{1}{\Gamma(\alpha)} \int_{0}^{x}(x-t)^{\alpha-1} f(t) d t \\
= & \frac{1}{\Gamma(\alpha)} \int_{x}^{1}(1-t)^{\alpha-1} f(t) d t x^{\alpha-1} \\
& +\frac{1}{\Gamma(\alpha)} \int_{0}^{x}\left((x-x t)^{\alpha-1}-(x-t)^{\alpha-1}\right) f(t) d t .
\end{aligned}
$$

Clearly, for any $x \in D$, the first integral is nonnegative. Similarly, $(x-x t)^{\alpha-1}>$ $(x-t)^{\alpha-1}$ holds for all $t \in(0, x)$, and thus the second integral is also nonnegative. Hence, $T f \in K$, i.e., the operator $T$ is positive. Now it follows directly from the Krein-Rutman theorem [6, Theorem 19.2] that the spectral radius of $T$ is an eigenvalue of $T$, and an eigenfunction $u \in K \backslash\{0\}$.

\section{ACKNOWLEDGEMENTS}

The research of B. Jin and W. Rundell was supported by US NSF Grant DMS1319052, R. Lazarov was supported in part by US NSF Grant DMS-1016525 and J. Pasciak was supported by NSF Grant DMS-1216551. The work of all authors was supported also by Award No. KUS-C1-016-04, made by King Abdullah University of Science and Technology (KAUST).

\section{REFERENCES}

[1] R. A. Adams and J. J. F. Fournier, Sobolev Spaces, 2nd ed., Pure and Applied Mathematics (Amsterdam), vol. 140, Elsevier/Academic Press, Amsterdam, 2003. MR2424078 (2009e:46025) 
[2] Q. M. Al-Mdallal, An efficient method for solving fractional Sturm-Liouville problems, Chaos Solitons Fractals 40 (2009), no. 1, 183-189, DOI 10.1016/j.chaos.2007.07.041. MR2517924 (2010f:65133)

[3] I. Babuška and J. Osborn, Eigenvalue problems, Handbook of Numerical Analysis, Vol. II, Handb. Numer. Anal., II, North-Holland, Amsterdam, 1991, pp. 641-787. MR.1115240

[4] D. A. Benson, S. W. Wheatcraft, and M. M. Meerschaert, The fractional-order governing equation of Lévy motion, Water Resour. Res. 36 (2000), no. 6, 1413-1424.

[5] A. S. Chaves, A fractional diffusion equation to describe Lévy flights, Phys. Lett. A 239 (1998), no. 1-2, 13-16, DOI 10.1016/S0375-9601(97)00947-X. MR1616103 (99a:82067)

[6] K. Deimling, Nonlinear Functional Analysis, Springer-Verlag, Berlin, 1985. MR787404 $(86 \mathrm{j}: 47001)$

[7] D. del-Castillo-Negrete, Chaotic transport in zonal flows in analogous geophysical and plasma systems, Phys. Plasmas 7 (2000), no. 5, 1702-1711, DOI 10.1063/1.873988. 41st Annual Meeting of the Division of Plasma Physics of the American Physical Society (Seattle, WA, 1999). MR.1784245(2001f:76021)

[8] D. del-Castillo-Negrete, B. A. Carreras, and V. E. Lynch, Front dynamics in reactiondiffusion systems with Levy flights, Phys. Rev. Lett. 91 (2003), (1):018302, 4 pp.

[9] D. del-Castillo-Negrete, B. A. Carreras, and V. E. Lynch, Nondiffusive transport in plasma turbulence: a fractional diffusion approach, Phys. Rev. Lett. 94 (2005), (6):065003, 4 pp.

[10] M. M. Djrbashian, Harmonic Analysis and Boundary Value Problems in the Complex Domain, Operator Theory: Advances and Applications, vol. 65, Birkhäuser Verlag, Basel, 1993. Translated from the manuscript by H. M. Jerbashian and A. M. Jerbashian [A. M. Dzhrbashyan]. MR 1249271 (95f:30039)

[11] N. Dunford and J. T. Schwartz, Linear Operators. I. General Theory, With the assistance of W. G. Bade and R. G. Bartle. Pure and Applied Mathematics, Vol. 7, Interscience Publishers, Inc., New York; Interscience Publishers, Ltd., London, 1958. MR0117523(22 \#8302)

[12] M. M. Džrbašjan, A boundary value problem for a Sturm-Liouville type differential operator of fractional order (Russian, with Armenian and English summaries), Izv. Akad. Nauk Armjan. SSR Ser. Mat. 5 (1970), no. 2, 71-96. MR0414982 (54 \#3074)

[13] A. Ern and J.-L. Guermond, Theory and Practice of Finite Elements, Applied Mathematical Sciences, vol. 159, Springer-Verlag, New York, 2004. MR2050138 (2005d:65002)

[14] V. J. Ervin and J. P. Roop, Variational formulation for the stationary fractional advection dispersion equation, Numer. Methods Partial Differential Equations 22 (2006), no. 3, 558-576, DOI 10.1002/num.20112. MR 2212226(2006m:65265)

[15] V. J. Ervin and J. P. Roop, Variational solution of fractional advection dispersion equations on bounded domains in $\mathbb{R}^{d}$, Numer. Methods Partial Differential Equations 23 (2007), no. 2, 256-281, DOI 10.1002/num.20169. MR2289452(2007k:65147)

[16] R. Gorenflo, J. Loutchko, and Y. Luchko, Computation of the Mittag-Leffler function $E_{\alpha, \beta}(z)$ and its derivative, Fract. Calc. Appl. Anal. 5 (2002), no. 4, 491-518. MR.1967847 (2004d:33020a)

[17] R. Gorenflo, J. Loutchko, and Y. Luchko, Correction: "Computation of the Mittag-Leffler function $E_{\alpha, \beta}(z)$ and its derivative" [Fract. Calc. Appl. Anal. 5 (2002), no. 4, 491-518; MR1967847 (2004d:33020a)], Fract. Calc. Appl. Anal. 6 (2003), no. 1, 111-112. MR1992470 (2004d:33020b)

[18] P. Grisvard, Elliptic Problems in Nonsmooth Domains. Pitman, Boston, MA, 1985.

[19] B. Jin, R. Lazarov, J. Pasciak, and W. Rundell, A finite element method for the fractional Sturm-Liouville problem, preprint, available as arXiv:1307.5114, 2013.

[20] B. Jin and W. Rundell, An inverse Sturm-Liouville problem with a fractional derivative, J. Comput. Phys. 231 (2012), no. 14, 4954-4966, DOI 10.1016/j.jcp.2012.04.005. MR2927980

[21] A. A. Kilbas, H. M. Srivastava, and J. J. Trujillo, Theory and Applications of Fractional Differential Equations, North-Holland Mathematics Studies, vol. 204, Elsevier Science B.V., Amsterdam, 2006. MR2218073 (2007a:34002)

[22] J.-L. Lions and E. Magenes, Problèmes aux limites non homogènes et applications. Vol. 1 (French), Travaux et Recherches Mathématiques, No. 17, Dunod, Paris, 1968. MR0247243 (40 \#512)

[23] R. Metzler and J. Klafter, The random walk's guide to anomalous diffusion: a fractional dynamics approach, Phys. Rep. 339 (2000), no. 1, 77, DOI 10.1016/S0370-1573(00)00070-3. MR:1809268(2001k:82082) 
[24] A. M. Nahušev, The Sturm-Liouville problem for a second order ordinary differential equation with fractional derivatives in the lower terms (Russian), Dokl. Akad. Nauk SSSR 234 (1977), no. 2, 308-311. MR0454145 (56 \#12396)

[25] I. Podlubny, Fractional Differential Equations: An introduction to fractional derivatives, fractional differential equations, to methods of their solution and some of their applications, Mathematics in Science and Engineering, vol. 198, Academic Press, Inc., San Diego, CA, 1999. MR $1658022(99 \mathrm{~m}: 26009)$

[26] I. Podlubny, A. Chechkin, T. Skovranek, Y. Chen, and B. M. Vinagre Jara, Matrix approach to discrete fractional calculus. II. Partial fractional differential equations, J. Comput. Phys. 228 (2009), no. 8, 3137-3153, DOI 10.1016/j.jcp.2009.01.014. MR2509311(2010c:65144)

[27] A. Yu. Popov and A. M. Sedletskiǔ, Distribution of roots of Mittag-Leffler functions (Russian), Sovrem. Mat. Fundam. Napravl. 40 (2011), 3-171, DOI 10.1007/s10958-013-1255-3; English transl., J. Math. Sci. (N. Y.) 190 (2013), no. 2, 209-409. MR2883249(2012k:33049)

[28] S. G. Samko, A. A. Kilbas, and O. I. Marichev, Fractional Integrals and Derivatives: Theory and Applications, Gordon and Breach Science Publishers, Yverdon, 1993. MR 1347689 (96d:26012)

[29] A. H. Schatz, An observation concerning Ritz-Galerkin methods with indefinite bilinear forms, Math. Comp. 28 (1974), 959-962. MR0373326 (51 \#9526)

[30] H. Seybold and R. Hilfer, Numerical algorithm for calculating the generalized Mittag-Leffler function, SIAM J. Numer. Anal. 47 (2008/09), no. 1, 69-88, DOI 10.1137/070700280. MR2452852(2009i:30024)

[31] C. Shen and M. S. Phanikumar, An efficient space-fractional dispersion approximation for stream solute transport modeling, Adv. Water Res. 32 (2009), no. 10, 1482-1494.

[32] M. Kh. Shkhanukov, On the convergence of difference schemes for differential equations with a fractional derivative (Russian), Dokl. Akad. Nauk 348 (1996), no. 6, 746-748. MR1440740 (97m:65135)

[33] T. H. Solomon, E. R. Weeks, and H. L. Swinney, Observation of anomalous diffusion and Lévy flights in a two-dimensional rotating flow, Phys. Rev. Lett. 71 (1993), no. 24, 3975-3978.

[34] E. Sousa, Finite difference approximations for a fractional advection diffusion problem, J. Comput. Phys. 228 (2009), no. 11, 4038-4054, DOI 10.1016/j.jcp.2009.02.011. MR.2524510 (2010e:65134)

[35] C. Tadjeran, M. M. Meerschaert, and H.-P. Scheffler, A second-order accurate numerical approximation for the fractional diffusion equation, J. Comput. Phys. 213 (2006), no. 1, 205-213, DOI 10.1016/j.jcp.2005.08.008. MR2203439 (2006j:65253)

[36] H. Wang and T. S. Basu, A fast finite difference method for two-dimensional spacefractional diffusion equations, SIAM J. Sci. Comput. 34 (2012), no. 5, A2444-A2458, DOI 10.1137/12086491X. MR3023711

[37] H. Wang and D. Yang, Wellposedness of variable-coefficient conservative fractional elliptic differential equations, SIAM J. Numer. Anal. 51 (2013), no. 2, 1088-1107, DOI 10.1137/120892295. MR 3036999

[38] X. Zhang, M. Lv, J. W. Crawford, and I. M. Young, The impact of boundary on the fractional advection-dispersion equation for solute transport in soil: Defining the fractional dispersive flux with the caputo derivatives, Adv. Water Res. 30 (2007), no. 5, 1205-1217.

Department of Mathematics, University of California, Riverside, 900 University Ave., Riverside, California 92521

Current address: Department of Computer Science, University College London, Gower Street, London WC1E 6BT, United Kingdom

E-mail address: bangti.jin@gmail.com 3368

Department of Mathematics, Texas A\&M University, College Station, Texas 77843-

Department of Mathematics, Texas A\&M University, College Station, Texas 778433368

Department of Mathematics, Texas A\&M University, College Station, Texas 778433368

E-mail address: lazarov,pasciak,rundell@math.tamu.edu 